\title{
Concentration Gradient-Based Soft Robotics: Hydrogels Out of Water
}

Antonio López-Díaz ${ }^{\dagger}$, Ana Martín-Pacheco ${ }^{\dagger}$, Antonio M. Rodríguez, M. Antonia Herrero, Andrés S. Vázquez*, Ester Vázquez*

$\dagger$ These authors contributed equally to this work.

A. López-Díaz, Prof. A. S. Vázquez.

Escuela Técnica Superior de Ingeniería Industrial, Universidad de Castilla-La Mancha, 13071 Ciudad Real, Spain.

E-mail: andress.vazquez@uclm.es

Dr. A. Martín-Pacheco, Dr. A. M. Rodríguez, Prof. M. A. Herrero, Prof. E. Vázquez. Instituto Regional de Investigación Científica Aplicada (IRICA), Universidad de Castilla-La Mancha, 13071 Ciudad Real, Spain.

Facultad de Ciencias y Tecnologías Químicas, Universidad de Castilla-La Mancha, 13071 Ciudad Real, Spain.

E-mail: ester.vazquez@uclm.es

Keywords: hydrogels, soft robotics, actuators, graphene

Hydrogels are biocompatible soft materials that resemble biological tissues more than any other material. However, the use of these systems in soft robotics has been limited to aqueous environments. In the work published to date, hydrogels have relied on external water to swell or shrink in response to stimuli and, therefore, to actuate macroscopically.

In the work reported here, this limitation has been overcome by synthesizing a novel type of electroactive hydrogels capable of actuating when a low electric field is applied, even outside water. The bending actuation of these materials is caused by the movement of solvated ions within the hydrogel, which generates a concentration gradient, making it possible to use them directly in ambient-air conditions. A mathematical model for this behaviour is proposed. Issues like resistive heating and material drying have been addressed by preparing graphene hybrid hydrogels and by using hygroscopic salts.

Two applications are presented as a demonstration of the capabilities of these hydrogels: a soft gripper with two continuum actuators and a soft fingertip capable of changing its volume and stiffness. In addition, the possibility of fabrication by 3D printing technologies enhances the applicability of these promising materials, thus paving the way for innovative developments. 


\section{Introduction}

Plants move as a response to stimuli in a much simpler way than animals. In the latter, some proteins transform chemical energy into motion, whereas in plants the movement occurs by the osmotic flow of water due to concentration gradients. Hydrogels are a type of soft material known for their ability to mimic this simple mechanism of water uptake and release that takes place in plant cells. ${ }^{[1]}$ This behaviour makes them exciting tools for robotic applications.

In recent years, hydrogels have been developed as actuators, ${ }^{[2]}$ sensors, ${ }^{[3]}$ skins, ${ }^{[4]}$ manipulators, ${ }^{[5]}$ micro-robots, ${ }^{[6]}$ and bioinspired robots. ${ }^{[7]}$ The use of an external aqueous medium in which the hydrogel swells and shrinks ${ }^{[6,8]}$ has been crucial in every example of macroscopic actuation to date. However, plants do not have to be submerged in water to move, so the question arises as to the possibility of taking hydrogels out of water and inducing movement in response to a stimulus.

A number of hydrogels are able to respond to external stimuli such as $\mathrm{pH}$, light, temperature or electric fields. ${ }^{[9]}$ This property has opened many lines of research and has allowed these materials to be applied, not only as actuators in water but also in fields as varied as sensors or drug delivery systems. ${ }^{[10]}$ One of the most important qualities of these soft materials is that they can be custom-designed to form the biocompatible structures that have the Young moduli that most closely resemble biological tissues. ${ }^{[1]}$ The functional and mechanical properties of these materials can be tuned by changing the starting monomers or crosslinkers in the polymerization process or by adding fillers (e.g., carbon materials) to modify their properties. ${ }^{[12]}$

The possibility of using a material with all of the above characteristics is stimulating, especially because of the implications that such work may have with human interfacing devices ${ }^{[13]}$ and also because it can improve performance in deformable robotics systems. Soft robots are usually made from rubber-like materials actuated by tendons (formed by tension cables or shapememory alloy actuators) or by pneumatic systems. ${ }^{[14]}$ Pneumatic actuators need a pneumatic 
power supply system which makes the device bulky and even noisy and introduces rigid elements in the structure. In some cases, the material that forms the robot structure is also responsible for the actuation, or even the sensing part, thus facilitating the compactness, integrity and simplicity of the robot. These configurations have commonly been bioinspired by invertebrate animals ${ }^{[15]}$ or plants, ${ }^{[16]}$ as in the case reported here. In this manner, if one could make the material itself produce the action, it would be possible to develop continuous systems that could move in different positions without the need for their movements to be defined first - in contrast to conventional hard robots - thanks to the compliance of these systems. Consequently, the development of continuous systems greatly favours manufacturing and reduces operational costs.

The work described here involved the study of the macroscopic movements of a hydrogel as a result of the concentration gradients that occur inside the material. The motion occurs in response to an electric field and without the need for an external aqueous system to mediate the swelling. A mechanism and a model to describe the macroscopic changes are proposed. The material was applied to a fingertip with adjustable stiffness and the possible challenges, such as an increase in temperature as a consequence of resistive heating or material drying, were mitigated through the preparation of graphene hybrids and the use of hydroscopic salts.

The study constitutes a pioneering work, and the possibility of preparing these hydrogels by $3 \mathrm{D}$ printing technologies paves the way for new developments in the soft robot materials palette. ${ }^{[8 i]}$

\section{Results}

\subsection{Synthesis and characterization of materials. Preparation of graphene hybrid hydrogels}

The electroactive materials used in this work are based on the monomer [2(acryloyloxy)ethyl] trimethylammonium chloride (AETA) (Figure 1a). A cationic electroactive 
network $(\mathrm{CN})$ was developed with a very high swelling degree and good mechanical properties. This material was prepared by radical photopolymerization of AETA as monomer and $N, N^{\prime}-$ methylenebisacrylamide $(M B A)$ as crosslinker using deionized water as solvent (see Experimental Section for details on the synthesis). The material was polymerized using UV light in conjunction with sodium (2,4,6-Trimethylbenzoyl)phenylphosphinate (NaTPO) as a photoinitiator (see Supporting Information for its synthesis). The use of NaTPO allowed an increase in the initiator concentration because of its high solubility in water in comparison to other photoinitiators. ${ }^{[17]}$ The higher concentration of photoinitiator also reduced the reaction time from nine hours (thermal polymerization) ${ }^{[18]}$ to a few seconds, thus allowing the use of 3D printing fabrication.

Graphene hybrid hydrogels $(\mathrm{CNG})$ were synthesized in order to ascertain whether this nanomaterial could improve the performance of our initial hydrogel (see Experimental Section for details regarding the synthesis). Graphene-based hydrogels have received attention in recent years due to the ability of the nanomaterial to enhance the hydrogel mechanical and electrical properties. ${ }^{[19]}$ Graphene can take an active part in the polymerization reaction acting as a covalent crosslinker in the polymeric network. To prepare a homogeneous hybrid hydrogel, it is important to have stable suspensions of graphene flakes in water, the solvent in which the polymerization takes place. However, this is difficult to achieve because graphene is a hydrophobic material. The easiest way to suspend graphene in water is to prepare graphene oxide, but this material no longer has the excellent conductive properties of graphene. We previously reported a straightforward way to prepare few-layer graphene (FLG) dispersions in water, by a mechanochemical approach using a ball milling treatment (see Supporting Information for more details). ${ }^{[20]}$ Despite being soluble in water, this graphene material has very few defects, it is not oxidized (as evidenced by its characterization, see Supporting Information and Figure S1) and it gives low resistivity values $(5.40 \pm 0.07 \Omega / \square)$, i.e., much lower than those 
observed for graphene oxide $(4.11-6.67 \mathrm{M} \Omega / \square)^{[21]}$ or reduced graphene oxide $(500-1100$ $\Omega / \square)^{[22]}$

The initial $\mathrm{CN}$ and $\mathrm{CNG}$ compositions are $50.50 \pm 0.74 \%$ (by weight, \% wt.) of water. Both materials can swell in an external aqueous medium, and the quantity of water contained in them can reach a maximum of $99.65 \pm 0.07 \%$ and $99.33 \pm 0.04 \%$, respectively. These levels of water correspond to a swelling degree $(S D)$ of $409 \pm 7$ for $\mathrm{CN}$ and $149 \pm 8$ for $\mathrm{CNG}$, as determined by means of a swelling degree study (Figure 1b) (see Supporting Information for the procedure for this study as well as for the equivalence between $S D$ and $\%$ wt. of water).

a)

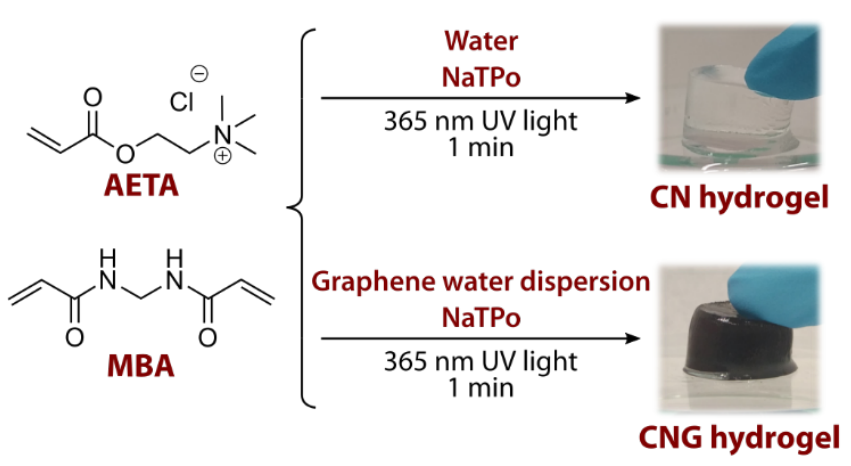

b)

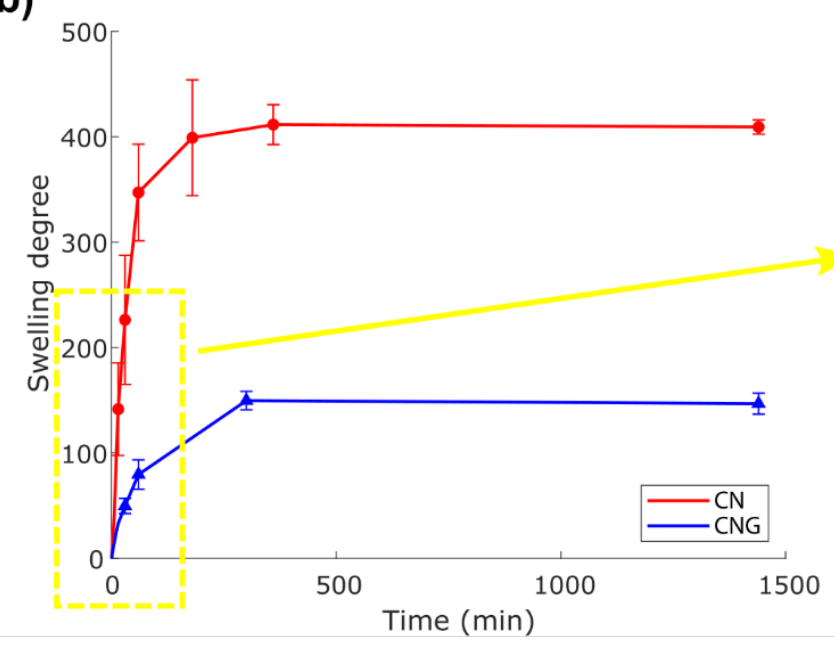

c)
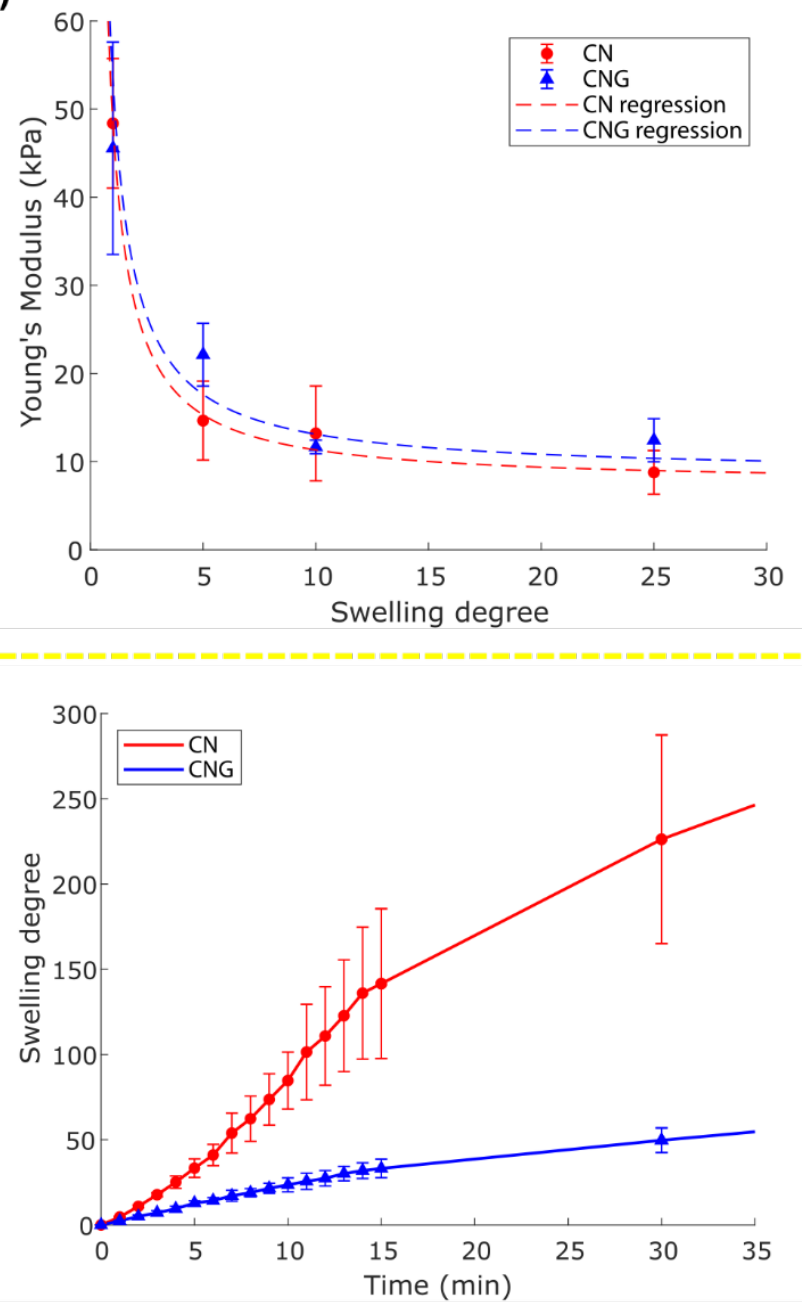

Figure 1. CN and CNG hydrogels, synthesis and properties. (a) Synthesis of CN and CNG hydrogels. (b) Swelling degree study for CN and CNG hydrogels. (c) Young's Modulus of $\mathrm{CN}$ and $\mathrm{CNG}$ hydrogels depending on the swelling degree. 
The average Young's Modulus values for $\mathrm{CN}$ and $\mathrm{CNG}$ hydrogels depending on the swelling degree are represented in Figure 1c (see Supporting Information for details). As expected, and as demonstrated in the literature, ${ }^{[23]}$ the higher the swelling degree, the lower the Young's Modulus of the material. Young's Modulus values for freshly prepared (1.02 swelling degree) $\mathrm{CN}$ and $\mathrm{CNG}$ are $48.4 \pm 7.3$ and $45.5 \pm 12 \mathrm{kPa}$, respectively. Thus, these two materials have comparable mechanical properties, together with a similar porous structure as observed by cryoScanning Electron Microscopy (cryo-SEM) (see Supporting Information and Figure S2).

\subsection{Response of the material under electric fields in different media}

\subsubsection{Aqueous medium}

The use of hydrogels in soft robotic applications, as proposed in related works, is based on their bending behaviour in poly ionic aqueous solutions when an electric field is applied. ${ }^{[8 c, 8 g, 24]}$ The response is caused by osmotic pressure changes that occur in the medium in the presence of an electric field. ${ }^{[8 a, 8 b, 25]}$ For example, in the case of a polyanionic structure, there is a decrease in the ionic concentration on the side close to the anode, which leads to the swelling of this zone. This change makes the hydrogel bend toward the cathode.

Given the mechanism outlined above, the bending behaviour of our materials was studied using a conductive aqueous solution $(0.05 \mathrm{M} \mathrm{LiCl})$ as the external medium. Hydrogels were also swollen in this medium at different swelling degrees, and these samples were analyzed to study the influence of the amount of water inside the material.

Three different tests were performed to study the bending behaviour of the hydrogel: Constant voltage test, in which $20 \mathrm{~V}$ was applied for 7 minutes; Voltage inversion test, in which $20 \mathrm{~V}$ was applied for 2.5 minutes and then $-20 \mathrm{~V}$ were applied for 3 minutes, thus inverting the polarity of the system; and Voltage cycles test, in which the polarity of the system was changed from $20 \mathrm{~V}$ to $-20 \mathrm{~V}$ every two minutes for around 10 minutes. 
The results of these studies show the temporal evolution of the curvature of the hydrogel $(K)$. This curvature, which is the inverse of the radius $(R)$ of the circumference drawn by the hydrogel, was obtained from images captured by a camera (Figure 2a and S3) (see Supporting Information for more details).

The response of hydrogels with different swelling degrees was tested, and the results are shown in Figure 2 (b, c and d). It can be observed that, in the constant voltage test (Figure 2b), the higher the swelling degree, the lower the curvature observed. This fact can be explained because of the swelling behaviour of the hydrogel: a hydrogel with $S D 2$ can absorb more water from the medium than a hydrogel with $S D 25$. Besides, this absorption at $S D 2$ is faster than at $S D$ 25 (see the swelling study of Figure 1b, where the slope of the curve is higher at lower $S D$ ). Therefore, the observed curvature at $S D 2$ is higher. In an effort to corroborate this finding, the mass of the hydrogel was controlled before and after the experiment, and it was found that the hydrogel with $S D 2$ experienced a mass gain of around $213.8 \pm 56.3 \%$, whereas the sample with SD 25 gained only $19.9 \pm 5.1 \%$. The entrance of water was significantly more intense for the hydrogel with $S D$ 2, and this probably caused the higher errors observed in comparison with those from $S D 9.3$ and 25.

Regarding the voltage inversion test (Figure 2c and Movie S1), the same behaviour as in the constant voltage test was observed prior to changing the polarity of the system. As one would expect, once the voltage was inverted $(\sim 150 \mathrm{~s})$, the hydrogels started to bend toward the anode, and the hydrogel with the lowest swelling degree bent faster and more than the others. This behaviour is also caused by the higher amount of water that this hydrogel can gain when compared to the others, as mentioned above.

Concerning the voltage cycles test (Figure 2d), for all samples, the bending effect was recovered in each cycle, albeit not completely. This behaviour could be explained because hydrogels swell when they are submerged in water even without applying a voltage, so, during the experiment, water enters into the hydrogels and, after several cycles, hydrogels no longer have $S D 2$ and 
they, therefore, become more slow-moving. The reversibility of the system is therefore compromised.

Comparison of $\mathrm{CNG}$ and $\mathrm{CN}$, at a lower $S D$, shows that the obtained curvature is slightly higher in the graphene hybrid hydrogel (Figure S4). This fact can be explained by a higher gain of water from the external medium during the experiment. In fact, on studying the swelling degree of $\mathrm{CNG}$ and $\mathrm{CN}$ in $\mathrm{LiCl}$ aqueous solutions (Figure $\mathbf{S 5}$ ), a faster entrance of water in $\mathrm{CNG}$ was observed during the first few minutes.
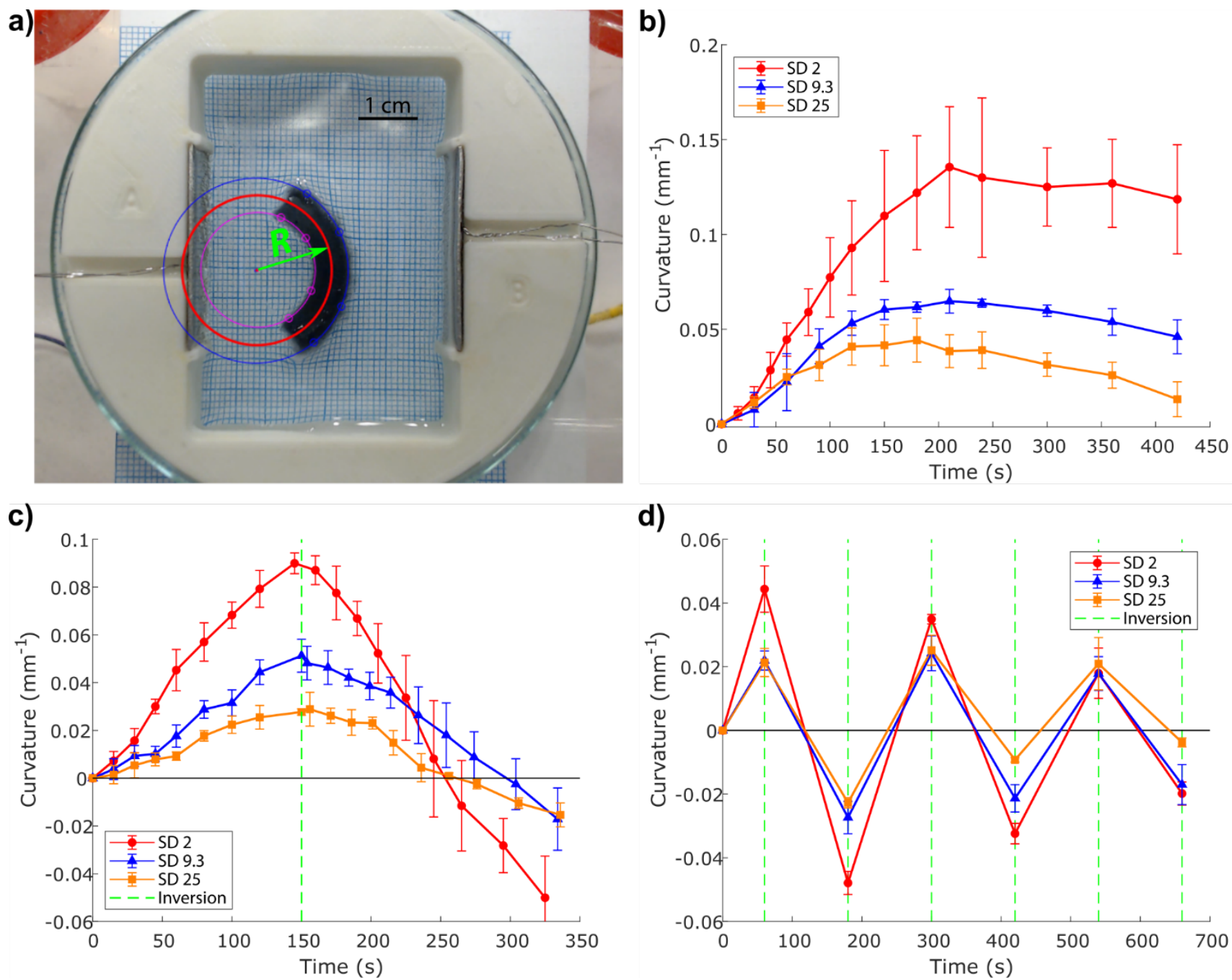

Figure 2. $\mathrm{CN}$ hydrogel under an electric field in an aqueous medium. (a) Example of an image captured by the camera during the tests: the radius of curvature $(R)$ is obtained from the edges and the mean circumference of the hydrogel. Results in an aqueous-based medium for CN hydrogel at different swelling degrees: (b) Constant voltage test, (c) Voltage inversion test and (d) Voltage cycles test. 


\subsubsection{Ionic liquid medium}

In order to ascertain whether our hybrid hydrogels were able to bend without swelling from an external aqueous medium, a method was developed to follow easily the movements of the ions inside the material. An ionic liquid (IL), namely 1-butyl-3-methylimidazolium tetrafluoroborate $\left(B M I M B F_{4}\right)$ (see Supporting Information for its synthesis), was used as an alternative conductive medium to water.

$\mathrm{BMIMBF}_{4}$ was selected not only because of its ionic conductivity, stability, and low melting point but also because $\mathrm{CN}$ and $\mathrm{CNG}$ do not swell in this medium and the drying effect of the hydrogels is prevented. Therefore, it can be ensured that the hydrogels do not interact with the external medium.

Remarkably, the application of an electric field to this material under these conditions made the hydrogel bend toward the cathode, in contrast to the behaviour observed on using an aqueousbased external medium (Figure 3a).

The first two tests described in the previous section were repeated under these new conditions. It was previously shown that the curvatures for $\mathrm{CN}$ and $\mathrm{CNG}$ hydrogels are comparable in the aqueous medium and therefore only the data for $\mathrm{CN}$ are shown. The results are represented in Figure 3b for the constant voltage test and in Figure 3c for the voltage inversion test (Movie S2). Under these conditions, a higher swelling degree led to a higher curvature of the hydrogels. This finding can be explained as being due to the higher amount of water inside the hydrogel, which could facilitate the movement of ions. The enhancement of the bending effect upon increasing the $S D$ was observed in both tests.

With the aim of studying in greater depth the movements of ions and the influence of the added mobile ions inside the hydrogel, the concentration of the solution of $\mathrm{LiCl}$ in which the hydrogel was swollen was varied. Thus, $\mathrm{CN}$ was swollen in a low concentration $\mathrm{LiCl}$ solution $(0.005 \mathrm{M})$ at $S D 25$, and the curvature of the hydrogel was analyzed using the IL as the conductive medium 
(Figure S6a). Comparable curvatures were obtained even with a lower concentration of free ions inside the hydrogel. These results suggest that the concentration of the added mobile ions does not modify the curvature of the hydrogel.

a)
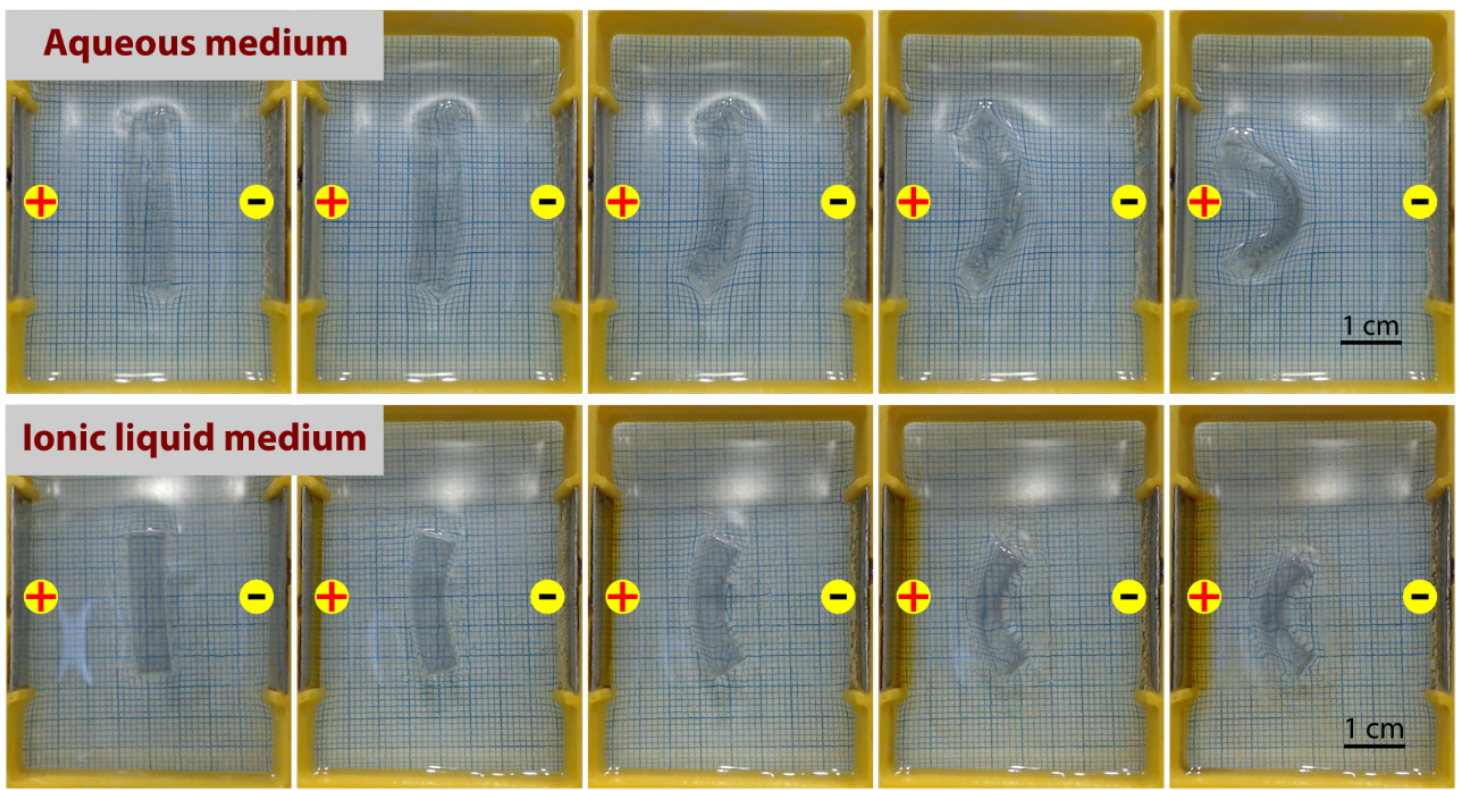

Time

b)

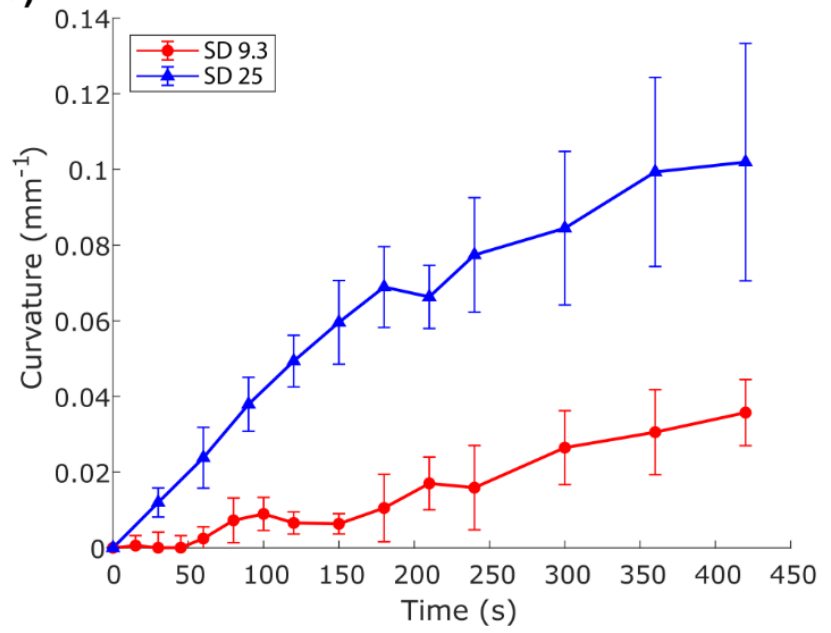

c)

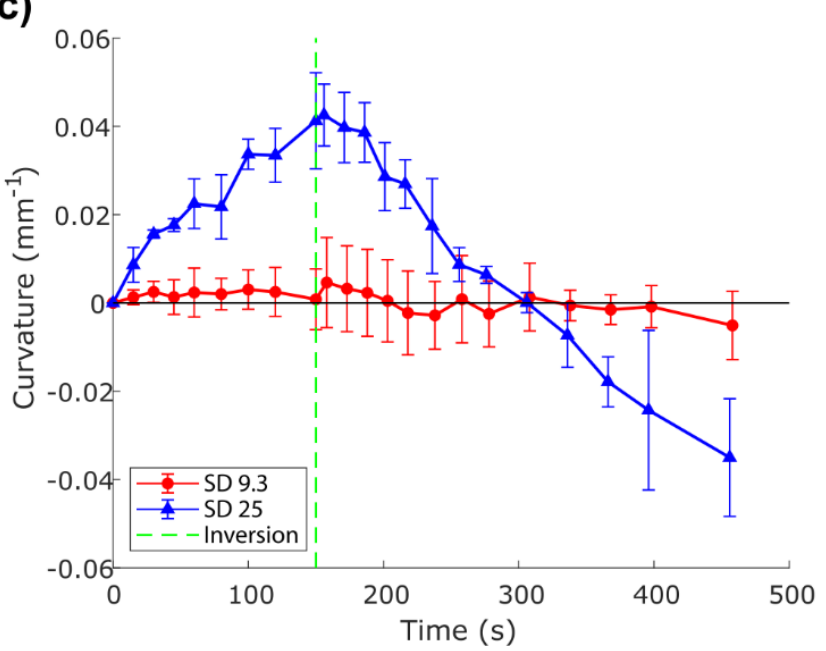

Figure 3. $\mathrm{CN}$ hydrogel under an electric field in an ionic liquid medium. (a) Time-lapse images of the bending behaviour of our hydrogels in $0.05 \mathrm{M} \mathrm{LiCl}$ aqueous solution (top) and ionic liquid medium (bottom). Results in an ionic liquid medium for $\mathrm{CN}$ hydrogel at different swelling degrees: (b) Constant voltage test and (c) Voltage inversion test.

The CN hydrogels were swollen with different inorganic salts to study the influence that the added mobile ions have on the curvature of the hydrogel by varying both the cation and the 
anion of the salt used. In this experiment, $\mathrm{LiBr}, \mathrm{LiClO}_{4}, \mathrm{KF}, \mathrm{KCl}$ and $\mathrm{KBr}$ were used at different concentrations. The selected inorganic salts allowed a comparison of the behaviour of the hydrogel on changing the cation and the anion and their respective sizes. The results show that the curvature experienced by the hydrogel was comparable regardless of the salt used and its concentration (Figure S6b and S6c), thus revealing the low influence of the added salt on the bending mechanism.

The results discussed above suggest that the added mobile ions do not play any role in the bending behaviour of the hydrogels. In an effort to corroborate this finding, the curvature of CN hydrogels swollen with deionized water was studied (Figure S6d, black line). The curvature obtained was comparable to that observed when mobile ions were incorporated in the hydrogel structure before the study, thus confirming that the bending behaviour depends on the mobile anions provided from the polymer network and not on the added mobile ions. It is worth noting that these extra ions were required in the experiments performed in aqueous-based media to obtain a conductive medium. Such extra ions are not required on using ionic liquids as a conductive medium.

The prepared hydrogel responds to an electric field by simply moving the solvated ions within the material and, as a consequence, it would be possible to produce the bending of the hydrogel without the use of an external ionic liquid-based medium by using flexible electrodes able to bend at the same time as the hydrogel. This possibility is discussed in the following section.

\subsubsection{Ambient-air conditions}

Aluminium foil was used to make flexible electrodes. Applying a $4 \mathrm{~V}$ electric field, it was possible to observe the bending of the hybrid hydrogels for the first time in ambient-air conditions, thus avoiding the use of an external medium (see Supporting Information for more details). As shown in the videos (Movie S3 and S4), both CN and CNG with $96.15 \%$ water 
( $S D$ 25) bend toward the cathode showing the same performance over time (Figure $\mathbf{S 7}$ for $\mathrm{CN}$ and Figure 4a for CNG).
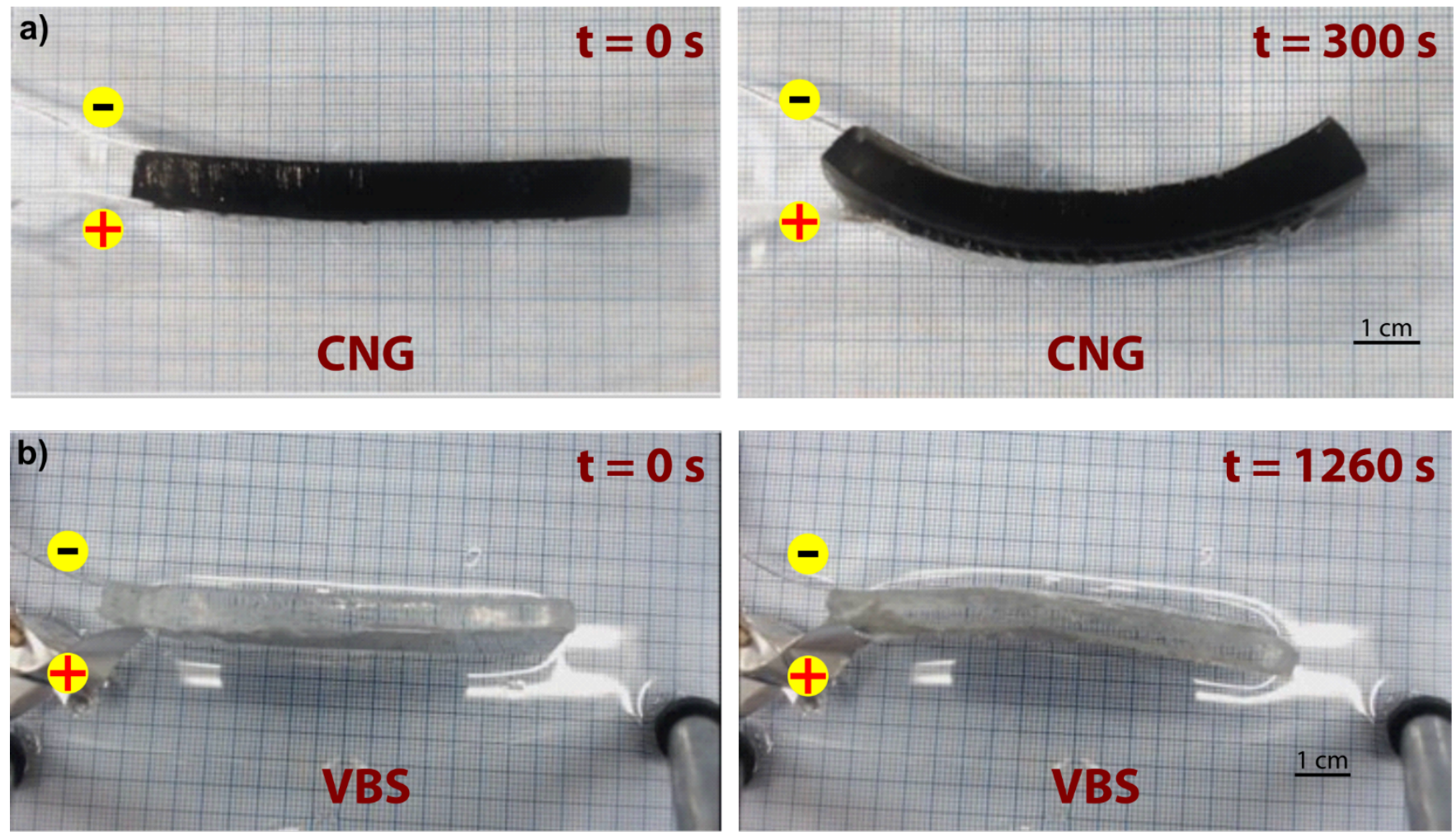

c)

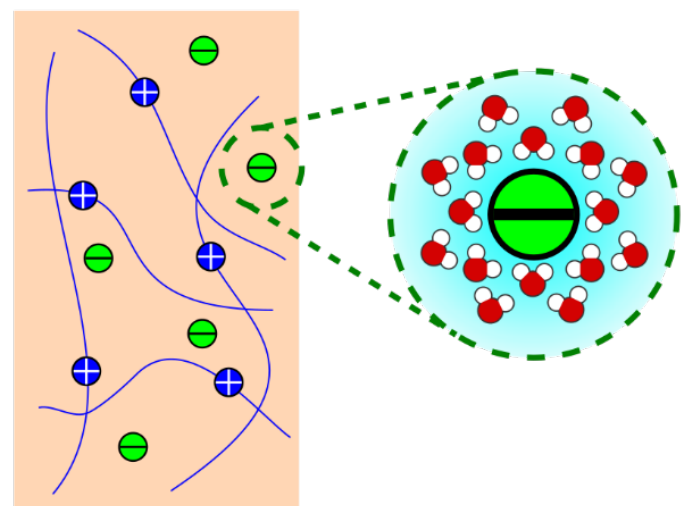

Hydrogel

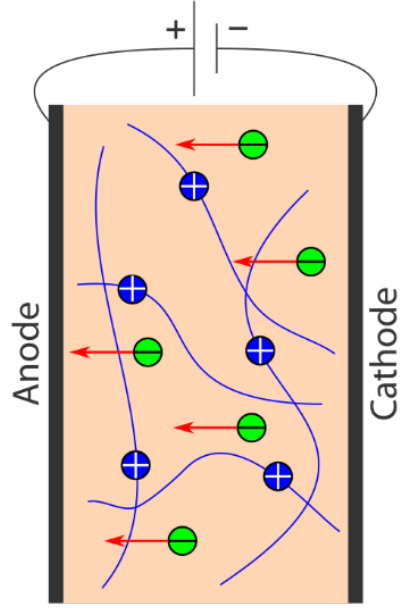

Applying electric field

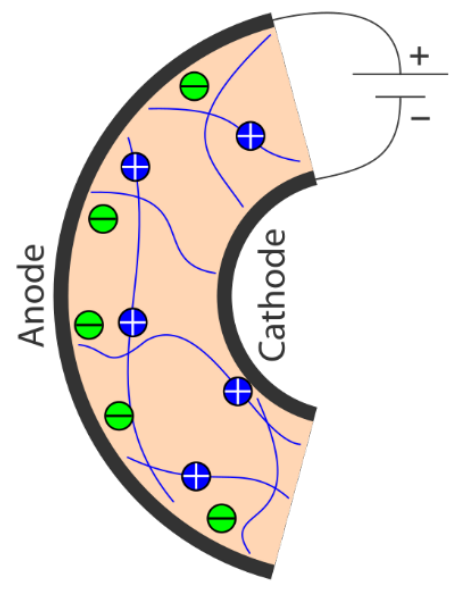

Swelling / Shrinking

Figure 4. Curvature of CNG and VBS hydrogels in ambient-air conditions and proposed mechanism diagram. (a) Curvature of CNG in ambient-air conditions. (b) Curvature of VBS in ambient-air conditions. (c) Schematic representation of a hydrogel with negative mobile ions and its bending response under an electric field. 
Moreover, a different hydrogel was studied to elucidate the role of the electroactive polymer network in the bending behaviour of the materials. This alternative was a 4vinylbenzenesulfonate (VBS)-based hydrogel, an anionic polymer network (see Experimental Section for synthesis) in which the mobile anions are $\mathrm{Na}^{+}$. The bending behaviour of the anionic hydrogel VBS is shown in Figure $4 \mathbf{b}$. In contrast to the case of $\mathrm{CN} / \mathrm{CNG}$, the VBS hydrogel bends toward the anode. Moreover, it is important to note that the bending of this material is not as effective and VBS requires more time to move than $\mathrm{CN} / \mathrm{CNG}$, thus demonstrating the important role of the counter-ion and the polymer network in the movement of our materials. Considering all of the experiments discussed above, in the following section, we propose a mechanism that leads to a mathematical model that explains the behaviour of our material.

\subsection{Mechanism of bending in ambient-air conditions and mathematical model}

Considering the previous results, the proposed mechanism for the bending behaviour is based on the movement of the mobile ions through the porous structure of the hydrogel when the electric field is applied. These ions drag the water in their solvation shells with them, thus giving rise to a gradient in the water-polymer proportion (i.e., swelling degree) inside the hydrogel. In the case of $\mathrm{CN} / \mathrm{CNG}$ hydrogel, the mobile ions are $\mathrm{Cl}^{-}$and the behaviour of this material is shown in Figure 4c. When the electric field is applied, free $\mathrm{Cl}^{-}$ions move towards the anode due to electrical forces. This change causes an increase in the amount of water (i.e., a swelling effect) in the region closest to the anode, while the amount of water decreases (i.e., a shrinking effect) in the region closest to the cathode. As a result, the hydrogel bends towards the cathode. This mechanism is consistent with the behaviour observed in the VBS hydrogel. In this case, the mobile ions are positive $\left(\mathrm{Na}^{+}\right)$and, as one would expect, the material bends towards the anode. It can be seen in Figure 4 ( $a$ and $b$ ) that the VBS bending process is much slower than the $\mathrm{CN} / \mathrm{CNG}$ bending. This behaviour occurs for three reasons: the lower mobility of $\mathrm{Na}^{+}$ions 
compared to $\mathrm{Cl}^{-}$ions ${ }^{[26]}$ and the smaller solvation shell of $\mathrm{Na}^{+[27]}$, together with a lower concentration of mobile ions in VBS when compared to CN (1.33 vs $5.25 \mathrm{M})$.

While this mechanism is similar to the one described for Ionic Polymer Metal Composites (IPMCs), ${ }^{[28]}$ to the best of our knowledge, no such behaviour has been described in hydrogels. IPMCs contain a small amount of hydrophilic ionic units intercalated into hydrophobic polymers which results in a heterogeneous system with less water uptake than in hydrogels. These two differences explain that, after an initial fast motion in IPMCs, a back relaxation takes place, as a consequence of contraction due to charge accumulation in very small hydrophilic domains. This back relaxation is not observed in our homogeneous materials.

Our materials are adaptable with a Young Modulus that resembles human organs such as heart and brain, which opens new possibilities in human interfacing devices. Moreover, hydrogels can be prepared with any shape and thickness without changing their properties, while IPMC actuators are only a few millimetres thick and attempts to increase the size reduce the water uptake and the bending is compromised. ${ }^{[29]}$ Finally, as we will show in section 2.5 , the stiffness changes observed in our materials, as a consequence of the electric fields, permit the application as fingertips, something that is not possible to achieve using IPMCs. The tuning of the stiffness helps to adjust the interaction with biological systems. Our compliant material could also be used for example to improve biomechanics acting as walking assistant or in wearable assistive devices. $^{[30]}$

On the basis of the above mechanism, a mathematical model is proposed here to predict the curvature of a hydrogel with certain species of mobile ions. The model proposed here has the same foundation as others, ${ }^{[31]}$ but our development is much simpler. This model is structured in two parts: firstly, an electrical part to describe the movement of ions inside the hydrogel, and secondly, a mechanical part to describe the macroscopic curvature of a hydrogel beam. 


\subsubsection{Model (part I) - Ions movement}

The movement of a certain species $i$ inside a medium is described by the Nernst-Planck equation:

$\vec{J}_{i}=-D_{i}\left(\vec{\nabla} c_{i}+z_{i} c_{i} f \vec{\nabla} \phi\right)$

where $\vec{J}_{i}$ is the flux of the species $i$ in the medium, $D_{i}$ is its diffusion coefficient, $c_{i}$ is its concentration (spatial-dependent), $z_{i}$ is its valence, $\phi$ is the electric potential and $f=F / R T$ is a constant defined by the Faraday constant $(F)$, the gas constant $(R)$ and the temperature $(T)$.

On the right side of Equation 1, the diffusive term $\left(-D_{i} \vec{\nabla} c_{i}\right)$ defines the tendency of the species $i$ to be homogeneously concentrated in the medium, while the migration term $\left(-D_{i} z_{i} c_{i} f \vec{\nabla} \phi\right)$ defines the electrical attraction over the species $i$ depending on whether it is charged $\left(z_{i} \neq 0\right)$. In the type of hydrogel discussed here, the species $i$ is the mobile ion present in the hydrogel. If there are two (or more) species of mobile ions, each species has its own equation.

Following the development of the model, if there is no generation or consumption of the species $i$, which is the case here, a mass balance can convert Equation 1 into:

$$
\frac{\partial c_{i}}{\partial t}=\vec{\nabla}\left[D_{i}\left(\vec{\nabla} c_{i}+z_{i} c_{i} f \vec{\nabla} \phi\right)\right]
$$

This equation can be transformed into a one-dimensional partial differential equation (PDE) since the electric field is applied in a particular direction $(y)$ in this case (Figure 5a):

$$
\frac{\partial c_{i}}{\partial t}=\frac{\partial}{\partial y}\left(D_{i} \frac{\partial c_{i}}{\partial y}+D_{i} z_{i} c_{i} f \frac{\partial \phi}{\partial y}\right)
$$


This PDE can be solved (i.e., to obtain $\left.c_{i} \equiv c_{i}(y, t)\right)$ with the appropriate initial and boundary conditions, which reflect some features or considerations of our case (see Supporting Information for details).

Once Equation 3 is solved for each of the species of mobile ions, the water concentration distribution inside the hydrogel $(C W(y, t))$ can be estimated:

$$
C W(y, t)=\frac{\alpha_{f} C_{f 0}+\sum_{i} \alpha_{i} c_{i}(y, t)}{\alpha_{f} C_{f 0}+\sum_{i} \alpha_{i} C_{i 0}} C W_{0}
$$

where $c_{i}(y, t)$ are the solutions of Equation 3 for each species. $C W_{0}$ is the initial homogeneous concentration of water in the hydrogel and $C_{f 0}$ represents the uniform concentration of charges bound to the polymer network (constant value in time), while $C_{i 0}$ is the initial uniform concentration of each species. The coefficients $\alpha_{i}$ represent the percentage of water solvated in each kind of mobile ion, while $\alpha_{f}$ represents the percentage of water anchored to the network. The model described above allows an estimation of the water concentration distribution with respect to time $(C W(y, t))$, and this will be used in the mechanical model to predict the curvature of a hydrogel beam.

\subsubsection{Model (part II) - Bending}

The bending behavior of our hydrogel is very similar to that studied by Timoshenko, ${ }^{[32]}$ in which a bi-metallic strip was heated uniformly. The difference in the coefficients of expansion of the layers causes the material to bend as a whole.

The origin of the volumetric changes that occur in our materials is the change in the water distribution, not the temperature. However, the Timoshenko model for bi-layer materials can 
also be applied in this case, since this model considers the volumetric changes regardless of the causes. The Timoshenko model has the following expression for the curvature $(K)$ :

$$
K=\frac{1}{R}=\frac{\delta_{2}-\delta_{1}}{\frac{h}{2}+\frac{2\left(E_{1} I_{1}+E_{2} I_{2}\right)}{h}\left(\frac{1}{E_{1} a_{1}}+\frac{1}{E_{2} a_{2}}\right)}
$$

where subindexes denote the layer, $h$ is the total height of the whole strip, $a_{j}$ is the height of each layer (Figure 5a), $E_{j}$ is the Young's Modulus, $I_{j}$ is the planar moment of inertia and $\delta_{j}$ is:

$$
\delta_{j} \approx \frac{1}{3} \frac{\Delta V_{j}}{V_{j 0}}, \quad j=1,2
$$

in which $\Delta V_{j}$ is the volume change of layer $j$ and $V_{j 0}$ its initial volume.

In the hydrogel case, as the water distribution is previously obtained (Equation 4), these volume changes can be computed and, finally, the curvature of the strip (see Supporting Information for more details about this calculation).

Considering the whole model, with known values like the applied voltage $(V)$, the dimensions of the hydrogel $(h, w, L)$ and the initial concentration of the different species and water $\left(C_{f 0}, C_{i 0}\right.$, $\left.C W_{0}\right)$, and with other values $\left(D_{i}, \alpha_{i}, \alpha_{f}\right)$ estimated, a water concentration distribution is obtained $(C W(y, t))$. This result is used in conjunction with other known values $(E)$ to compute the curvature with respect to time $(K(t))$, which is the finality of the model.

\subsubsection{Model verification}

The model was tested, and the results were compared with the experimental response of $\mathrm{CN}$ and VBS specimens used to prove the bending under ambient-air conditions (Figure 5b). The 
curvatures of these specimens (red line for $\mathrm{CN}$ and blue line for VBS) were recorded with a camera (setup in Figure S3), while the model (orange line for CN and pink line for VBS) was run through a simulation with the parameters specified in Table S1. As explained above, some of these parameters, e.g., diffusion coefficients and $\alpha_{i}$ values, were estimated (see Supporting Information for more details).

a)

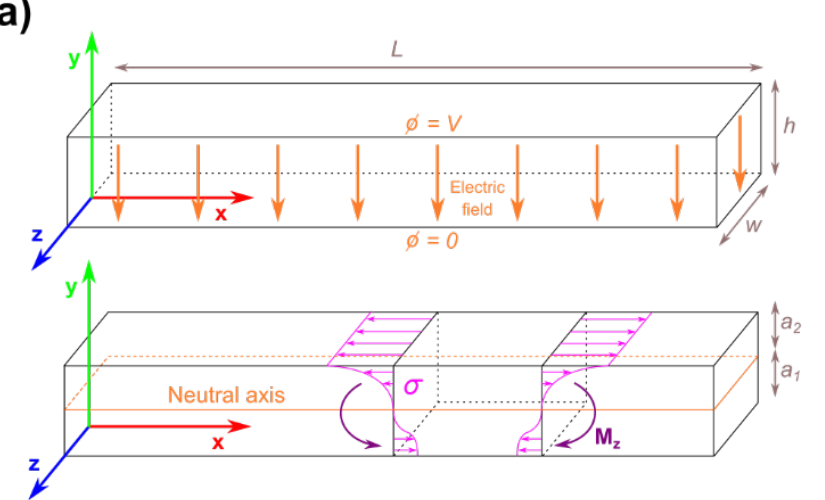

b)
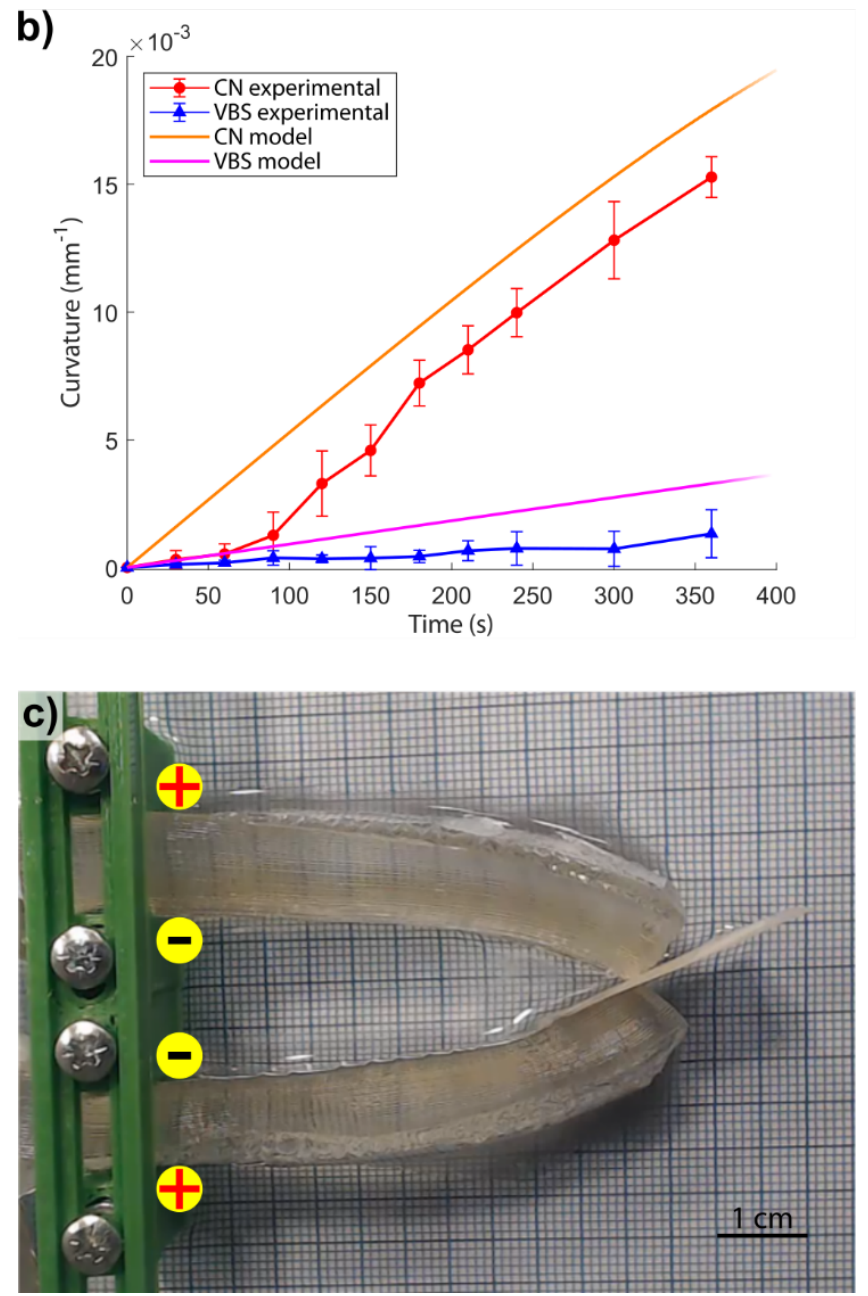
Figure 5. Parameters and verification of the bending mathematical model and gripper demonstrator. (a) Coordinate system and parameters used in the model. (b) Curvatures of CN and VBS hydrogels in ambient-air conditions and model simulations (curvatures are in absolute value, as CN and VBS bend towards opposite sides). (c) Simple gripper based on two continuum $\mathrm{CN}$ hydrogel actuators.

In both cases, the trend for the model is very similar to the experimental response. The good numerical performance of the model supports the mechanism described above. The influence of a greater number of mobile ions in $\mathrm{CN}$ than in VBS, together with the fact that those in $\mathrm{CN}$ carry more water, is noticeable through the model execution.

However, there are differences between the experimental responses and the model simulations. These discrepancies could be due to some minor effects that were neglected during the development of the model. For instance, the interaction between mobile particles and their influence on the electric potential or the interphase effect between the hydrogel and the electrodes. Consideration of all the known effects would have led to a more accurate but much more complicated model, ${ }^{[31]}$ so we decided to avoid this situation. It can be seen from the results that our model is reasonably simple but still manages to reproduce the hydrogel dynamics to an acceptable level.

\subsection{Gripper demonstrator: continuous bending as a response to electric fields}

The ambient-air actuation of the $\mathrm{CN}$ hydrogels can be used to design a soft gripper demonstrator with two continuum fingers that are able to grasp objects as a response to an electric field (Figure 5c and Movie S5).

The two soft fingers are made of the $\mathrm{CN}$-based actuators previously presented (section 2.2.3). These actuators are clamped from their ends by a 3D printed part designed for this purpose, and this setup leads to an effective actuator length of $\sim 60 \mathrm{~mm}$. The separation distance between the actuators is $\sim 30 \mathrm{~mm}$, and their transversal section is $\sim 10 \times 15 \mathrm{~mm}$, in which the shortest 
dimension corresponds to the distance between the electrodes in each actuator. As previously described, the electrodes are based on aluminium foil, and each hydrogel strip is actuated at its own voltage, which provides the gripper with two degrees of freedom (DOF).

The working principle of this gripper is simple: to close the fingers, the polarity of the voltage must be positive on the outside and negative on the inside due to the nature of $\mathrm{CN}$ bending, as discussed above. This closure operation takes about 3-4 minutes upon applying $4 \mathrm{~V}$ on each actuator with the gripper configuration described above. The opposite operation (i.e., to open the fingers) can be achieved by reversing the polarity of the electric field. This reversibility is guaranteed if the actuators do not lose the water contained within them.

Thanks to the two DOF and the model, each finger can be position-controlled independently, thus making the gripper more useful. For instance, this arrangement would allow the selection of the contact point between the fingers or the movement of an object already grasped.

\subsection{Fingertip demonstrator: electrically responsive volume and stiffness. Decrease in resistive heating}

As previously shown, $\mathrm{CN}$ hydrogels respond to an electric stimulus through variations in their swelling distribution. These changes lead to bending behaviour, but these hydrogels can also be used for other purposes like changing the volume or the stiffness of the material. ${ }^{[33]}$

If the electrodes are placed at the farthest ends of the hydrogel beam, and the electric field is applied in the longitudinal direction, the resultant mechanical effect is not bending. In this situation (Figure 6a), the geometry prevents the bar from bending, and the volume changes along the hydrogel beam become more visible. Furthermore, since a different swelling implies a different Young's Modulus (Figure 1c), the hydrogel beam experiences changes in stiffness. These volume and stiffness changes can be useful in robotic manipulation: soft fingertips are used for power grasps while hard fingertips are used for precision tasks. Increasing the volume 
of the fingertip is useful to allow the fingertip to conform to the shape of the object once it is grasped. Our $\mathrm{CN}$ hydrogel can be used for this purpose. In the region closest to the anode, the hydrogel swells and its stiffness decreases, while the opposite behaviour occurs in the region closest to the cathode. The idea of a CN-based fingertip is to use only one half of the hydrogel as the effective contact surface of the fingertip so that its volume or stiffness could be controlled by adjusting the applied electric field.

a)

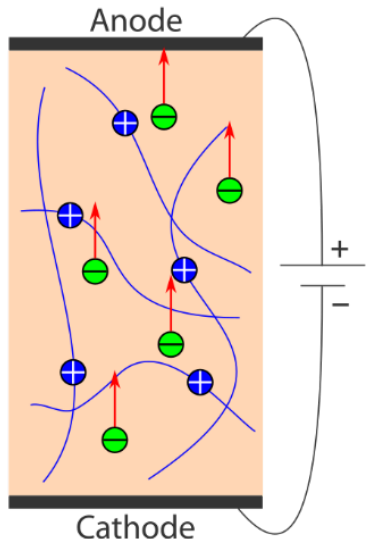

Applying electric field

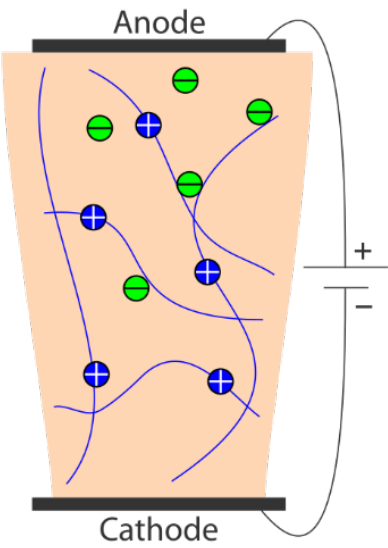

Swelling / Shrinking b)

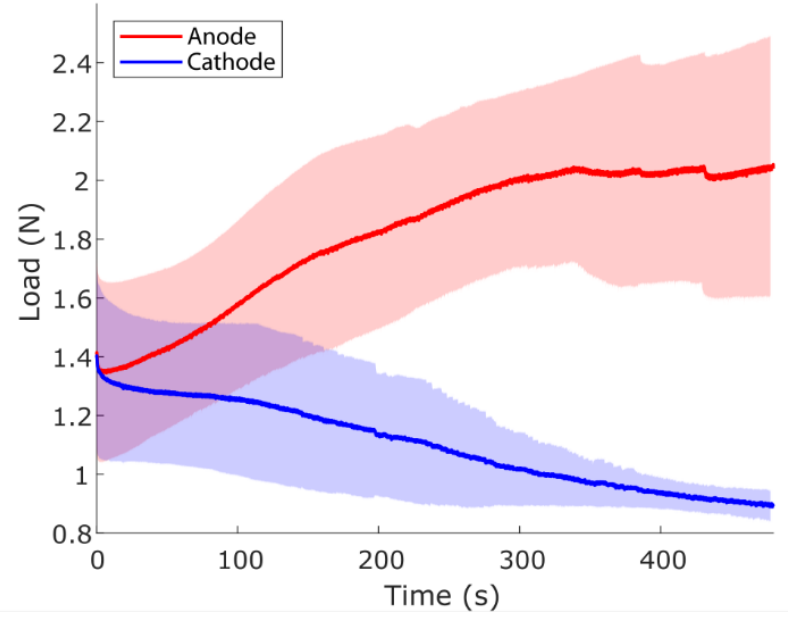

d)

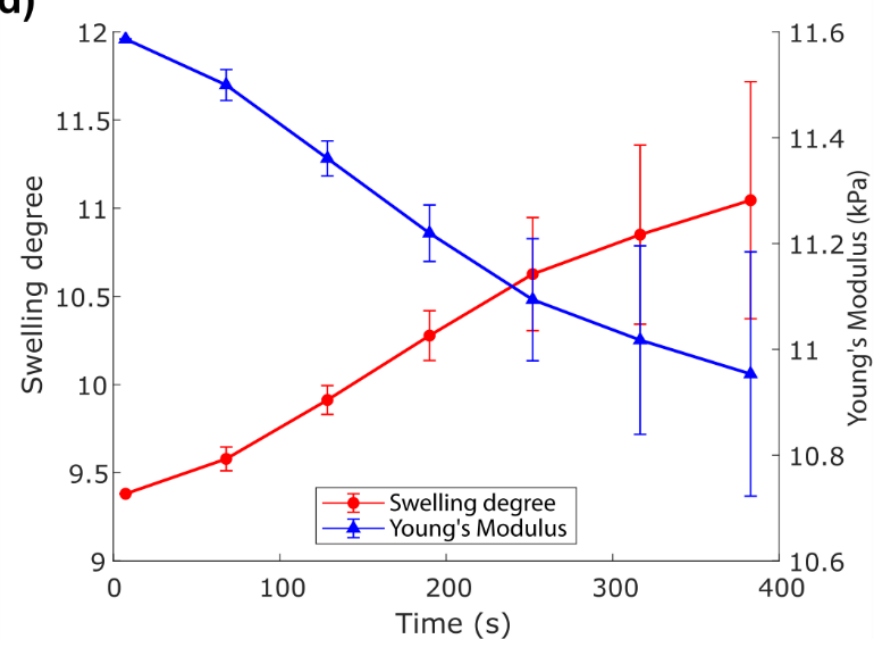

Figure 6. Fingertip mechanism and results of the volume and stiffness tests. (a) Schematic representation of a hydrogel with positive and negative mobile ions and its response under an electric field applied longitudinally. (b) Load change experienced by each half of the fingertip as the electric field is applied (shaded regions represent the errors). (c) Load change experienced by each half of the fingertip as the electric field is applied, with polarity inversion (shaded regions represent the errors). (d) Stiffness change experienced by the fingertip as the electric field is applied (region closest to the anode). 
Instead of using water, the hydrogel was swollen in $0.05 \mathrm{~m}$ aqueous $\mathrm{LiCl}$. This is one of the most hygroscopic salts known, and so, although we have already shown that salts are not required to produce movements in the hydrogel, $\mathrm{LiCl}$ was used to prevent the fingertip from drying out. ${ }^{[34]}$

In an effort to demonstrate the volume changes in the two halves of the hydrogel, the setup shown in Figure S8 was built. The test (see details in Supporting Information) attempts to replicate the consequences of changing the volume while an object is held. The half with the increasing volume is expected to exert higher forces with time. In contrast, the half with a decreasing volume will exert a reduced force. This response can be seen in Figure $6 \mathbf{b}$, while Figure 6c illustrates that the hydrogel shows a quick response upon reversing the gradient of the force immediately after the change in the polarity of the electric field.

In this manner, after grasping an object, the local force exerted by the $\mathrm{CN}$-based fingertip can be adjusted by controlling the applied voltage. However, the stiffness of the contact zone before touching the object can also be tuned, since changes in the swelling degree lead to changes in the Young's Modulus of the material.

As the correlation between swelling degree and Young's Modulus was shown previously, this stiffness-change is demonstrated through the changes in the height of the fingertip. A change in the height of the analyzed zone infers that swelling or deswelling has occurred and, therefore, Young's modulus has changed. The setup described above was used again but, in this case, to measure the height. The results of a test performed on the zone closest to the anode are shown

\section{in Figure 6d.}

The knowledge discussed above was used to develop a fingertip that was capable of changing its stiffness and volume (Figure 7a). However, there are some problems with this system, such as the material drying or heating as a consequence of the electric current, which also favours drying. The drying issue was addressed by swelling the hydrogel with a lithium chloride solution instead of deionized water, as explained above, while the potential heating problem 
was addressed by synthesizing graphene hybrid hydrogels (CNG). A CNG-based fingertip performs in the same manner as a $\mathrm{CN}$-based one, since $\mathrm{CNG}$ and $\mathrm{CN}$ have nearly the same Young's modulus (Figure 1c) and their electro-responses are the same, as stated in section 2.2.3. The only difference between a $\mathrm{CN}$ or CNG-based fingertip is the thermal behaviour.

Graphene has been widely reported to be an excellent thermal conductor ${ }^{[35]}$. Prior to this work, we already proved the improvement in heat dissipation by adding graphene to other hydrogel materials. ${ }^{[19 a]}$ Thus, the thermal properties of graphene would make CNG hydrogels less thermally resistive (i.e., they would heat up to a lesser extent) than $\mathrm{CN}$ hydrogels.

The above hypothesis was demonstrated by performing temperature tests on $\mathrm{CN}$ and $\mathrm{CNG}$ fingertip samples. These tests consisted of applying the same electric field to $\mathrm{CN}$ and $\mathrm{CNG}$ hydrogel fingertips and recording the temperatures with a thermal camera (Figure 7b) (see Supporting Information for more details and setup, Figure S9).

Three points in the fingertip were considered (Figure S9): on the side closest to the anode (P1), in the middle of the fingertip (P2) and on the side closest to the cathode (P3).

The evolution of the temperature at P1 and P2 when an electric field was applied is shown in Figure S10, while the evolution for P3 is shown in Figure 7c. At each point of the fingertip, the temperature increase was greater for $\mathrm{CN}$ than for $\mathrm{CNG}$. Nevertheless, the difference between $\mathrm{CN}$ and $\mathrm{CNG}$ was more evident at $\mathrm{P}$, where the material reached the highest temperature (Figure $7 \mathrm{~b}$ ) because this point is located on the shrinking side of the fingertip (Figure $6 \mathrm{a})$. At the end of the test ( $\sim 6 \mathrm{~min})$ the temperature difference between $\mathrm{CN}$ and $\mathrm{CNG}$ was $4{ }^{\circ} \mathrm{C}$ at $\mathrm{P} 3$, thus demonstrating the better temperature dissipation in the presence of graphene.

The influence of graphene in decreasing the thermal resistance of the samples was demonstrated. A CNG-based fingertip would heat up less than a CN-based fingertip, which would ultimately cause a lower loss of water and better performance of the system. 
a)

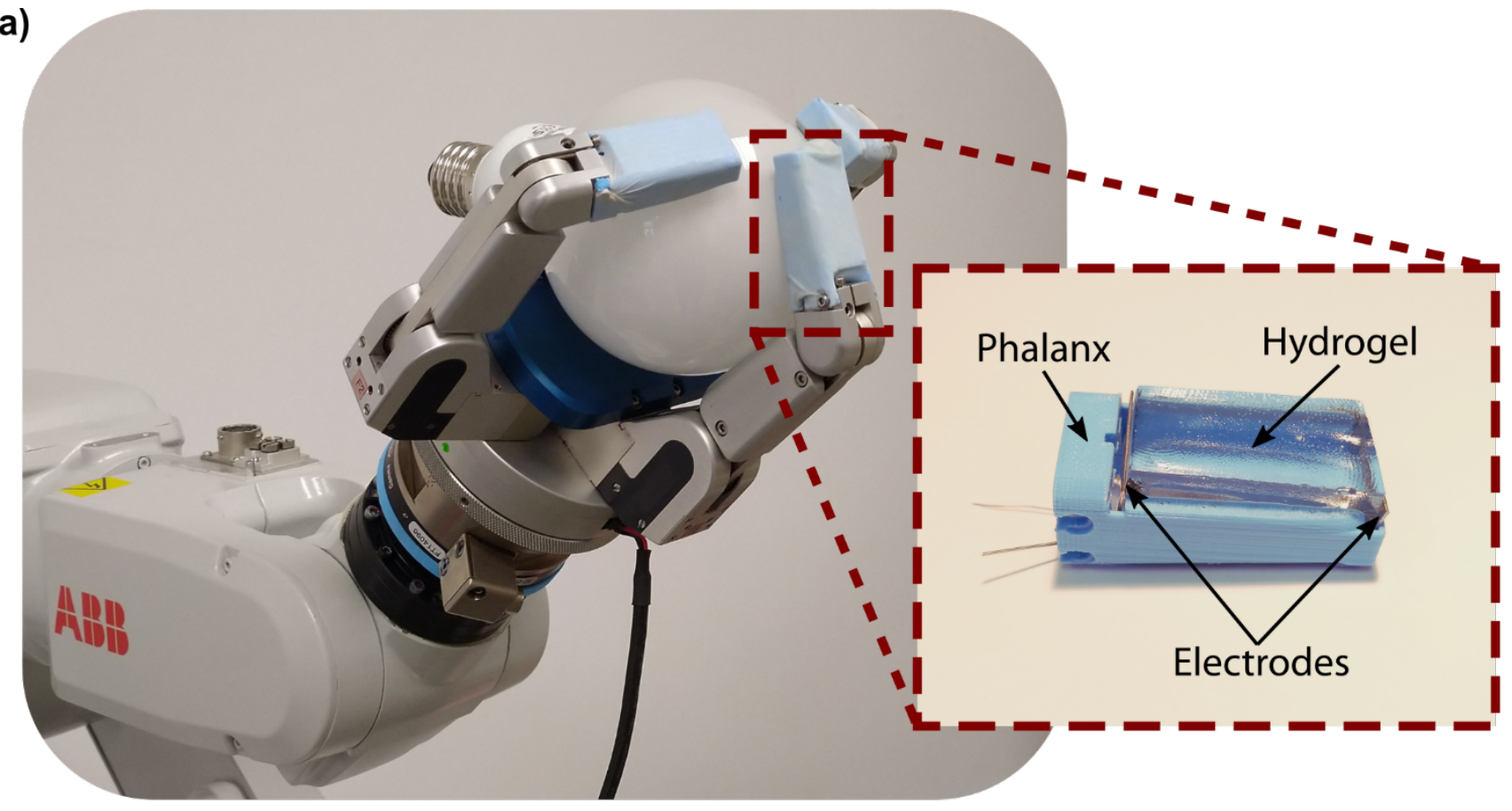

b)

c)
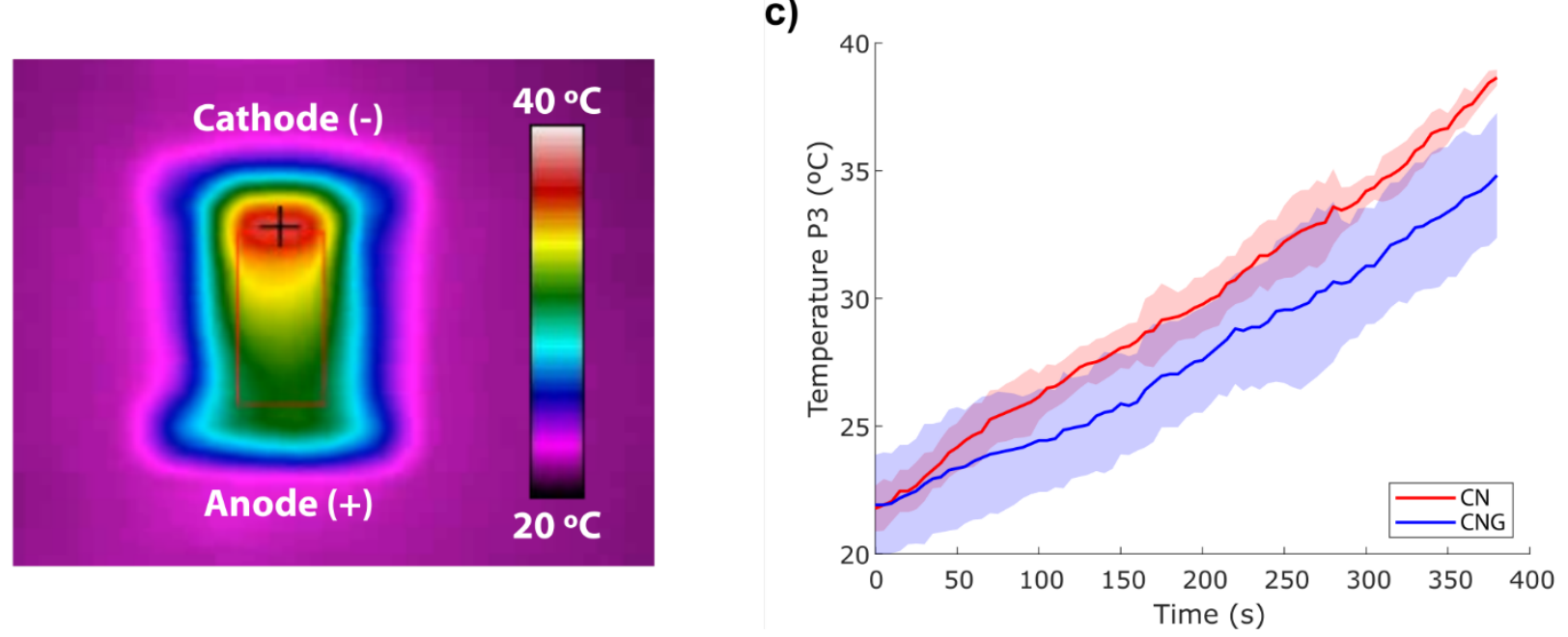

Figure 7. Fingertip prototype and thermal performance of the fingertips. (a) Hydrogel-based fingertip installed on a Barrett Hand. (b) Thermal image of the hydrogel fingertip. (c) Evolution of the temperature near the cathode in $\mathrm{CN}$ and $\mathrm{CNG}$ fingertips.

\subsection{D printing fabrication}

Finally, in an effort to increase the applicability of these materials, we considered the preparation of the hydrogels using 3D printing methodologies. This technology has been extensively used in soft robotics and has spread the design of sophisticated and complex geometries using a wide variety of materials in an environmentally friendly manner. ${ }^{[36]}$ 
For these applications, new formulations of hydrogel inks need to meet the following requirements: flow under modest pressures, gel quickly, and maintain sufficient integrity after the printing process. ${ }^{[37]}$ Inkjet, light-assisted, and extrusion-based 3D printing systems are the most suitable methods for hydrogel printing. ${ }^{[38]}$

The classical approach to designing a hydrogel precursor ink is to formulate a polymer solution that forms a network immediately after printing via different methods assisted by temperature, $\mathrm{pH}$, ion concentration, chemical activation or UV light. However, there are a limited number of suitable hydrogels that can act as inks and tuning their properties remains a challenge. ${ }^{[39]}$ The ability to photopolymerize our $\mathrm{CN}$ hydrogels in a few minutes led us to prepare them by $3 \mathrm{D}$ printing. However, the low viscosity did not allow these materials to be used to build a 3D structure in any controlled shape or enable the formation of more than two stacked layers of hydrogel (Figure S11a). Pre-polymerization of the $\mathrm{CN}$ ink was not a viable option since hydrogel formation is very quick.

The 3D printer employed was based on the Fused Deposition Modeling (FDM) technique (Figure 8a, see Supporting Information for more details). The reason for this choice instead of other 3D printing technologies, such as stereolithography, is the possibility of including more extruders and printing different materials at the same time. The working viscosity range of our printer is $0.003-6 \cdot 10^{4} \mathrm{~Pa} \cdot \mathrm{s}$ and its original purpose were for $3 \mathrm{D}$ printing of a chocolate-based ink. For this reason, the printer was adapted with a UV light system formed by four LEDs in order to polymerize the hydrogels (Figure 8b, see Supporting Information for more information). Chocolate was the original raw material for this printer, and its rheology, therefore, became a good reference point in search of an appropriate hydrogel ink: the viscosities for 3D printing are in the range of $10-1000 \mathrm{~Pa} \cdot \mathrm{s}^{[40]}$ 


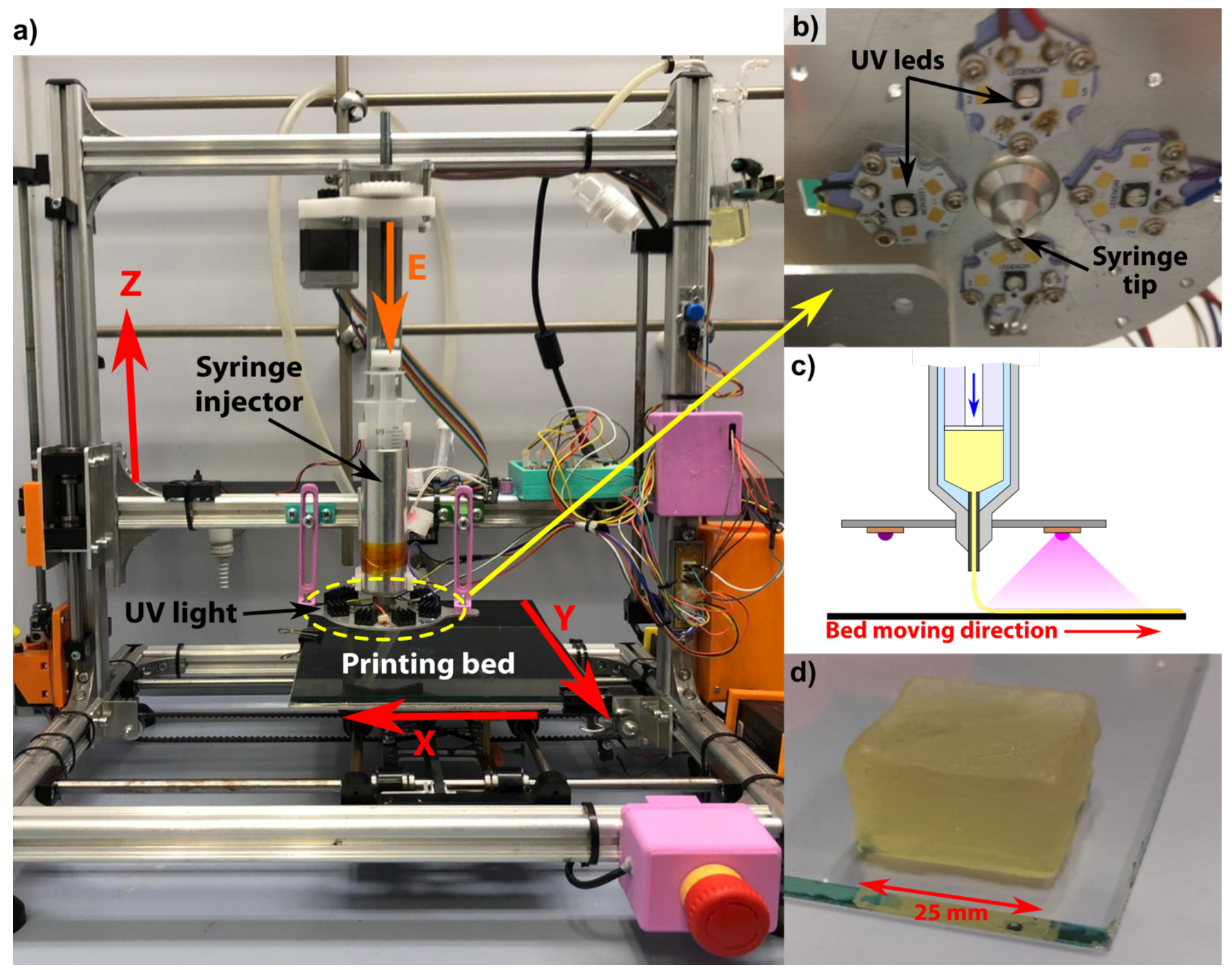

Figure 8. 3D printer, adaptations, operation and printed sample. (a) 3D printer adapted for our materials. (b) UV LED ring mounted around the syringe tip. (c) Schematic diagram of the directional switching of the LEDs. (d) 3D printed cube of a CN-ALG hydrogel.

\subsubsection{Rheology studies}

Given the problem outlined in the previous section, a further strategy was explored to improve the rheology of the hydrogel ink. This involved the addition of a thickening agent (also called a thixotropic agent) that would not affect the polymerization of the hydrogel. Sodium alginate was selected since it is a versatile polysaccharide, and its gelation properties have been widely explored. ${ }^{[41]}$ Examples of these alginate-based hydrogels have proven to be extremely tough, and they have mainly been used for bone or cartilage repair applications. ${ }^{[42]}$ 
Different concentrations of alginate solutions were prepared, and their rheological properties were compared with those of the pristine $\mathrm{CN}$ ink. The preparation and selection of the $\mathrm{CN}$ ink that included alginate (CN-ALG ink) was directed by the way in which the different alginate solutions extruded on the $3 \mathrm{D}$ printer. Under the preoptimized $3 \mathrm{D}$ printing conditions, the printing process worked much more smoothly, and complex shapes could be printed with the $25 \mathrm{mg} \cdot \mathrm{ml}^{-1}$ alginate solution. As a consequence, the CN-ALG solution was prepared with the same alginate concentration (see Supporting Information). For the sake of comparison, the zeroshear viscosity of the different solutions was measured by steady-state shear flow at very low shear stress (Figure S11b and Table S2).

The viscosity values of the different samples confirmed our initial observations on the $\mathrm{CN}$ ink. The $\mathrm{CN}$ ink viscosity $\left(3.99 \pm 0.06 \cdot 10^{-3} \mathrm{~Pa} \cdot \mathrm{s}\right)$ is at the lower range that is appropriate for our $3 \mathrm{D}$ printer, and this would explain the printing problems encountered. The CN-ALG ink has similar rheological behaviour to the $25 \mathrm{mg} \cdot \mathrm{ml}^{-1}$ solution of alginate $(5.54 \pm 0.27 v s 4.66 \pm 0.11 \mathrm{~Pa} \cdot \mathrm{s}$, respectively), which indicates that the presence of the $\mathrm{CN}$ hydrogel precursors did not have a negative impact on the rheology of the ink. The polymerization time for the hydrogel was also unchanged in the presence of the alginate (see Supporting Information for more details).

\subsubsection{Printing results}

In the $3 \mathrm{D}$ printing process, the main problem was the coordination of the UV polymerization jointly with the material extrusion. Given the rapid polymerization of the hydrogel, there were severe problems with blockages in the syringe tip. This was partly due to the high reflection of the UV light on the glass substrate (which allows good adhesivity of the ink) and the working disposition of the four UV LEDs (Figure 8b), which initially worked all at once. The high reflection could be solved by placing a dark sheet underneath the printing glass substrate. To solve the problem of blockage when the four UV LEDs were working at the same time, 
automatic and directional control of the LEDs was implemented on the printer controller. This new feature only allowed the LEDs to be switched on in the X-Y direction in which the printer was extruding, thus allowing instant irradiation of the extruded material only (Figure 8c). In this manner, only one or two LEDs were working at the same time and this significantly reduced the irradiation of the syringe tip.

Finally, with the UV LEDs working alternately, the 3D printing procedure worked much more smoothly and it was possible to optimize the different printing parameters, such as layer thickness, filling or printing speed. The optimized parameters for this type of hydrogel and shapes were $100 \%$ filling, $0.5 \mathrm{~mm}$ layer thickness, and $40 \mathrm{~mm} \cdot \mathrm{s}^{-1}$ printing speed. It can be seen (Figure 8d, Movie S6) that a freshly prepared CN-ALG ink enabled the fabrication of cubes with good definition and excellent polymerization.

The CN-ALG ink provided hydrogels that were tough enough to be 3D printed in various shapes without requiring a support material. The mechanical characterization of the $\mathrm{CN}-\mathrm{ALG}$ hydrogel showed that its Young's Modulus was markedly higher than that of the pristine CN hydrogel (48.4 \pm 7.3 vs $153.43 \pm 27.55 \mathrm{kPa}$ for $\mathrm{CN}$ and $\mathrm{CN}-\mathrm{ALG}$, respectively).

Nevertheless, the electroactive movement of this hydrogel remained intact, since these CNALG hydrogels are swollen under the same conditions as those outlined previously for $\mathrm{CN}$, and the hydrogel presents similar electroactive behaviour in a similar manner and with similar timings (Figure S11c).

\section{Conclusion}

Hydrogels are increasingly considered to be an important class of materials with broadly tunable physicochemical properties in numerous applications, including biomedicine, soft electronics, sensors or actuators, amongst others. In particular, we have demonstrated that the hydrogels presented in this work can be used in soft robotic applications as actuators at room 
temperature and, more importantly, in ambient-air conditions, which is not a common environment for this kind of hydrogel applications.

We have successfully manipulated the water contained within the hydrogels by applying an electric field, which produces a concentration gradient that is translated into a measurable mechanical deformation. These measurements were the key to understanding the chemical behaviour of these hydrogels under an electric field, and they allowed us to propose a mechanism and develop a simple mathematical model. Despite the simplicity of the model, it can predict the bending performance of a hydrogel strip in an acceptable manner.

The findings made in this study were exploited by developing two soft robot demonstrators that are able to work in ambient-air conditions: a simple soft gripper formed by two hydrogel-based actuators and a soft fingertip capable of changing its volume and stiffness. The strength of both applications resides in the capability of the material to change its intrinsic properties, a valued feature in several fields. Furthermore, we succeeded in introducing different chemical modifications to the hydrogel to enhance its rheology without affecting its electroactive behaviour. These advances allowed us to successfully produce hydrogel structures by $3 \mathrm{D}$ printing.

For the sake of simplicity, the proposed mathematical model does not consider minor effects such as the interaction between particles at an atomic level, distortion in the electric field as a consequence of the mobile ions, weak interactions within the polymer network, or the behaviour of the hydrogel-electrode interphase. Therefore, there is room for improvement and more complex and accurate models. Nevertheless, the simplicity of the mathematical model gave us the means to implement automatic control of our actuators, which is essential for the usability of simple robotic devices. For instance, the automatic control of the curvature of the continuum actuators would have an immediate impact on the gripper, since it would allow to select the contact point between the fingers or enable the movement of grasped objects. 
Regarding the challenges related to the design of new hydrogels, there is plenty of scope from a chemical point of view for the synthesis of new monomers and new crosslinkers to enhance the performance of our current materials. The addition of different fillers or nanoparticles could also improve the material strength/response, which would lead to better prototypes than those presented in this work. We are also working on a chemical encapsulation of the hydrogels to minimize the loss of water ${ }^{[43]}$ in an effort to increase the reversibility of the system. These possible future advances, together with $3 \mathrm{D}$ printing for the production of complex hydrogel shapes, will increase the possibilities for the design of new robotic applications.

Based on the demonstration that our hydrogels can actuate in ambient-air conditions, we are convinced that this kind of material warrants renewed efforts to enable them as emerging new materials for soft robotics applications. We strongly believe that this work not only enhances the fundamental understanding of hydrogels, but also shifts the paradigm in the use of these soft structures in numerous applications, thus paving the way for novel developments and the creation of promising new materials.

\section{Experimental section}

Materials: graphite was purchased from Bray Carbon Inc. (graphite SP-1 powder) and was used without further purification.

Reagents such melamine, AETA, MBA, KPS, LiCl, $\mathrm{LiBr}, \mathrm{KF}, \mathrm{KCl}, \mathrm{KBr}$ and $\mathrm{LiClO}_{4},(2,4,6-$ Trimethylbenzoyl)phenylphosphine acid ethyl ester, sodium iodate, methyl ethyl ketone, bromobutane and 1-methyimidizole were purchased from Sigma-Aldrich; sodium tetrafluoroborate was purchased from TCI Chemicals, and sodium alginate was purchased from Acros. All reagents were used without any treatment or purification.

Hydrogel synthesis: the synthesis of the $\mathrm{CN}$ hydrogel was carried out by radical polymerization of the monomer [2-(acryloyloxy)ethyl]trimethylammonium chloride $(26.4 \mathrm{mmol})$ and the 
crosslinker $N, N^{\prime}$-methylenebisacrylamide $(0.06 \mathrm{mmol})$ in deionized water $(5 \mathrm{ml})$. Sodium (2,4,6-Trimethylbenzoyl)phenylphosphinate $(0.06 \mathrm{mmol})$ was used as a radical photoinitiator. ${ }^{[18,}$ 33]

The synthesis of the graphene-based hydrogel was carried out by the same procedure but using a FLG-water dispersion $\left(2 \mathrm{mg} \cdot \mathrm{ml}^{-1}\right)$ as solvent.

In both cases, the mixtures were sonicated until homogenization and then photopolymerized under UV light (365 $\mathrm{nm}$ wavelength, four LEDs $1200 \mathrm{~mW}$ of irradiation power each) for 1 minute. The polymerization took place in a silicon-based mold.

For 3D printing applications, the CN-ALG solution was prepared by mixing AETA (105.6 $\mathrm{mmol})$, the crosslinker MBA $(0.24 \mathrm{mmol})$ and $\mathrm{NaTPO}(0.24 \mathrm{mmol})$ as the radical photoinitiator. Sodium alginate ( $1 \mathrm{~g}$ ) was used as an additive. The solution was completed by adding deionized water $(20 \mathrm{ml})$ as solvent (40 $\mathrm{ml}$ total volume).

The VBS-hydrogels were prepared using sodium 4-vinylbenzenesulfonate $(1.33 \mathrm{mmol})$ as monomer, MBA $(0.08 \mathrm{mmol})$ as crosslinker, potassium persulfate $(0.015 \mathrm{mmol})$ as initiator and milli-Q water as solvent $(1 \mathrm{ml})$. The polymerization took place in an oven at $70{ }^{\circ} \mathrm{C}$ for 1.5 hours.

\section{Supporting Information}

Supporting Information is available from the Wiley Online Library or from the author.

\section{Acknowledgements}

We acknowledge help and collaboration of Dr. R. Fernández in experiments regarding stiffness and volume changes, A. Fraile in FLG synthesis and characterization, and J. M. Galindo and A. Naranjo in hydrogel synthesis. This work is supported by the EU Graphene Core 2 (785219) and Core 3 (881603) European Union (Flagship project), the Spanish Ministerio de Economía y Competitividad (Project CTQ2017-88158-R) and the Junta de Comunidades de Castilla-La Mancha (Project SBPLY/17/180501/000204). A.L.D. and A.M.P. gratefully acknowledge the Spanish Ministerio de Educación, Cultura y Deporte for their FPU grants (grants FPU14/01431 and FPU17/02617). Raman spectra were collected using a Renishaw InVia Reflex confocal spectrometer (FEDER-JCCM funding, UNCM13-1E-1663). HR-SEM images were measured using a GeminiSEM 500 from Zeiss coupled with a Quorum cryo-SEM (FEDER-JCCM funding, UNCM15-CE-2839).

Received: ((will be filled in by the editorial staff))

Revised: ((will be filled in by the editorial staff)) Published online: ((will be filled in by the editorial staff)) 


\section{References}

[1] L. Ionov, Adv. Funct. Mater. 2013, 23, 4555.

[2] a) H. Yuk, S. Lin, C. Ma, M. Takaffoli, N. X. Fang, X. Zhao, Nat. Commun. 2017, 8, 14230; b) A. K. Mishra, T. J. Wallin, W. Pan, P. Xu, K. Wang, E. P. Giannelis, B. Mazzolai, R. F. Shepherd, Science Robot. 2020, 5.

[3] C. Larson, B. Peele, S. Li, S. Robinson, M. Totaro, L. Beccai, B. Mazzolai, R. Shepherd, Science 2016, 351, 1071.

[4] Y. Yu, H. Yuk, G. A. Parada, Y. Wu, X. Liu, C. S. Nabzdyk, K. Youcef-Toumi, J. Zang, X. Zhao, Adv. Mater. 2019, 31, 1807101.

[5] D. Han, C. Farino, C. Yang, T. Scott, D. Browe, W. Choi, J. W. Freeman, H. Lee, ACS Appl. Mater. Interfaces 2018, 10, 17512.

[6] H. Li, G. Go, S. Y. Ko, J.-O. Park, S. Park, Smart Mater. Struct. 2016, 25.

[7] a) T. Li, G. Li, Y. Liang, T. Cheng, J. Dai, X. Yang, B. Liu, Z. Zeng, Z. Huang, Y. Luo, T. Xie, W. Yang, Sci. Adv. 2017, 3; b) S. R. Shin, B. Migliori, B. Miccoli, Y. C. Li, P. Mostafalu, J. Seo, S. Mandla, A. Enrico, S. Antona, R. Sabarish, T. Zheng, L. Pirrami, K. Zhang, Y. S. Zhang, K. T. Wan, D. Demarchi, M. R. Dokmeci, A. Khademhosseini, Adv. Mater. 2018, 30, 1704189; c) X. Liu, J. Liu, S. Lin, X. Zhao, Mater. Today 2020, 36, 102.

[8] a) T. Shiga, T. Kurauchi, J. Appl. Polym. Sci. 1990, 39, 2305; b) S. Y. Kim, H. S. Shin, Y. M. Lee, C. N. Jeong, J. Appl. Polym. Sci. 1999, 73, 1675; c) S. J. Kim, S. G. Yoon, Y. H. Lee, S. I. Kim, Polym. Int. 2004, 53, 1456; d) X. Zhang, C. L. Pint, M. H. Lee, B. E. Schubert, A. Jamshidi, K. Takei, H. Ko, A. Gillies, R. Bardhan, J. J. Urban, M. Wu, R. Fearing, A. Javey, Nano Lett 2011, 11, 3239; e) G. Stoychev, N. Puretskiy, L. Ionov, Soft Matter. 2011, 7; f) P. Techawanitchai, M. Ebara, N. Idota, T.-A. Asoh, A. Kikuchi, T. Aoyagi, Soft Matter. 2012, 8; g) D. Morales, E. Palleau, M. D. Dickey, O. D. Velev, Soft Matter. 2014, 10, 1337; h) W. J. Zheng, N. An, J. H. Yang, J. Zhou, Y. M. Chen, ACS Appl. Mater. Interfaces 2015, 7, 1758; i) Y. Yamamoto, K. Kanao, T. Arie, S. Akita, K. Takei, ACS Appl. Mater. Interfaces 2015, 7, 11002; j) C. Yang, Z. Liu, C. Chen, K. Shi, L. Zhang, X. J. Ju, W. Wang, R. Xie, L. Y. Chu, ACS Appl. Mater. Interfaces 2017, 9, 15758.

[9] O. Erol, A. Pantula, W. Liu, D. H. Gracias, Adv. Mater. Technol. 2019, 4.

[10] a) D. Buenger, F. Topuz, J. Groll, Prog. Polym. Sci. 2012, 37, 1678; b) S. Merino, C. Martin, K. Kostarelos, M. Prato, E. Vazquez, ACS Nano 2015, 9, 4686; c) J. Li, D. J. Mooney, Nat. Rev. Mater. 2016, 1.

[11] a) M. Jia, M. Rolandi, Adv. Health Mater. 2020, 9, e1901372; b) H. Banerjee, M. Suhail, H. Ren, Biomimetics 2018, 3, 15.

[12] Y. S. Zhang, A. Khademhosseini, Science 2017, 356.

[13] a) H. Wang, X. Ma, Y. Hao, Adv. Mater. Interfaces 2017, 4; b) S. Gong, L. W. Yap, B. Zhu, W. Cheng, Adv. Mater. 2020, 32, e1902278; c) B. Grigoryan, S. J. Paulsen, D. C. Corbett, D. W. Sazer, C. L. Fortin, A. J. Zaita, P. T. Greenfield, N. J. Calafat, J. P. Gounley, A. H. Ta, F. Johansson, A. Randles, J. E. Rosenkrantz, J. D. LouisRosenberg, P. A. Galie, K. R. Stevens, J. S. Miller, Science 2019, 364, 458; d) T. Wang, M. Wang, L. Yang, Z. Li, X. J. Loh, X. Chen, Adv. Mater. 2020, 32, 1905522.

[14] D. Rus, M. T. Tolley, Nature 2015, 521, 467.

[15] S. Kim, C. Laschi, B. Trimmer, Trends Biotechnol. 2013, 31, 287.

[16] a) E. W. Hawkes, L. H. Blumenschein, J. D. Greer, A. M. Okamura, Sci. Robot. 2017, 2; b) A. Sadeghi, A. Mondini, B. Mazzolai, Soft Robot. 2017, 4, 211.

[17] S. Benedikt, J. Wang, M. Markovic, N. Moszner, K. Dietliker, A. Ovsianikov, H. Grützmacher, R. Liska, J. Polym. Sci. Part A: Polym. Chem. 2016, 54, 473. 
[18] A. Martin-Pacheco, A. E. Del Rio Castillo, C. Martin, M. A. Herrero, S. Merino, J. L. Garcia Fierro, E. Diez-Barra, E. Vazquez, ACS Appl. Mater. Interfaces 2018, 10, 18192.

[19] a) A. Servant, V. Leon, D. Jasim, L. Methven, P. Limousin, E. V. Fernandez-Pacheco, M. Prato, K. Kostarelos, Adv. Health Mater. 2014, 3, 1334; b) C. Martin, A. MartinPacheco, A. Naranjo, A. Criado, S. Merino, E. Diez-Barra, M. A. Herrero, E. Vazquez, Nanoscale 2019, 11, 4822.

[20] J. M. Gonzalez-Dominguez, V. Leon, M. I. Lucio, M. Prato, E. Vazquez, Nat. Protoc. 2018, 13, 495.

[21] J. Li, R. Cui, Y. Chang, X. Guo, W. Gu, H. Huang, K. Chen, G. Lin, J. Dong, G. Xing, B. Sun, $R S C A d v$. 2016, 6, 58028.

[22] M. Savchak, N. Borodinov, R. Burtovyy, M. Anayee, K. Hu, R. Ma, A. Grant, H. Li, D. B. Cutshall, Y. Wen, G. Koley, W. R. Harrell, G. Chumanov, V. Tsukruk, I. Luzinov, ACS Appl. Mater. Interfaces 2018, 10, 3975.

[23] Y. D. Zaroslov, O. E. Philippova, A. R. Khokhlov, Macromolecules 1999, 32, 1508.

[24] C. Yang, W. Wang, C. Yao, R. Xie, X. J. Ju, Z. Liu, L. Y. Chu, Sci. Rep. 2015, 5, 13622.

[25] a) M. Homma, Y. Seida, Y. Nakano, J. Appl. Polym. Sci. 2001, 82, 76; b) L. Migliorini, T. Santaniello, Y. Yan, C. Lenardi, P. Milani, Sensors Actuat. B Chem. 2016, 228, 758.

[26] S. Koneshan, J. C. Rasaiah, R. M. Lynden-Bell, S. H. Lee, J. Phys. Chem. B 1998, 102, 4193.

[27] a) J. A. White, E. Schwegler, G. Galli, F. Gygi, J. Chem. Phys. 2000, 113, 4668; b) D. D. Kemp, M. S. Gordon, J. Phys. Chem. A 2005, 109, 7688.

[28] a) C. Jo, D. Pugal, I.-K. Oh, K. J. Kim, K. Asaka, Prog. Poly. Sci. 2013, 38, 1037; b) Y. Bahramzadeh, M. Shahinpoor, Soft Robot. 2014, 1, 38; c) M. Shahinpoor, K. J. Kim, Smart Materials and Structures 2001, 10, 819.

[29] S. Li, J. Yip, Polymers 2019, 11, 91.

[30] a) I. De Falco, M. Cianchetti, A. Menciassi, Bioinspir. Biomim. 2017, 12, 056008; b) Y. Li, Y. Maeda, M. Hashimoto, Int. J. Adv. Rob. Syst. 2015, DOI: 10.5772/61815; c) N. Yves Periard, G. Z. Pataky, N. D. Grivon, M. Y. R. C. Civet US Patent US20170348181A1, 2017.

[31] a) Z. Zhu, L. Chang, T. Horiuchi, K. Takagi, A. Aabloo, K. Asaka, J. Appl. Phys. 2016, 119; b) S. Nemat-Nasser, J. Y. Li, J. Appl. Phys. 2000, 87, 3321.

[32] S. Timoshenko, J. Opt. Soc. Am. 1925, 11, 233.

[33] A. López-Díaz, A. Martín-Pacheco, R. Fernández, A. M. Rodríguez, M. A. Herrero, E. E. Vázquez, A. S. Vázquez, 2019 IEEE - ICRA 2019, DOI: 10.1109/ICRA.2019.87941055126.

[34] K. Tian, J. Bae, S. E. Bakarich, C. Yang, R. D. Gately, G. M. Spinks, M. In Het Panhuis, Z. Suo, J. J. Vlassak, Adv. Mater. 2017, 29, 1604827.

[35] a) M. I. Katsnelson, Mater. Today 2007, 10, 20; b) N. Wang, M. K. Samani, H. Li, L. Dong, Z. Zhang, P. Su, S. Chen, J. Chen, S. Huang, G. Yuan, X. Xu, B. Li, K. Leifer, L. Ye, J. Liu, Small 2018, DOI: 10.1002/smll.201801346e1801346.

[36] a) T. J. Wallin, J. Pikul, R. F. Shepherd, Nat. Rev. Mater. 2018, 3, 84; b) J. Z. Gul, M. Sajid, M. M. Rehman, G. U. Siddiqui, I. Shah, K. H. Kim, J. W. Lee, K. H. Choi, Sci. Technol. Adv. Mater. 2018, 19, 243.

[37] M. Guvendiren, H. D. Lu, J. A. Burdick, Soft Matter. 2012, 8, 260.

[38] T. Billiet, M. Vandenhaute, J. Schelfhout, S. Van Vlierberghe, P. Dubruel, Biomaterials 2012, 33, 6020.

[39] P. Heidarian, A. Z. Kouzani, A. Kaynak, M. Paulino, B. Nasri-Nasrabadi, ACS Biomater. Sci. Eng. 2019, 5, 2688. 
[40] R. Karyappa, M. Hashimoto, Sci. Rep. 2019, 9, 14178.

[41] a) H. J. Lee, Y. B. Kim, S. H. Ahn, J. S. Lee, C. H. Jang, H. Yoon, W. Chun, G. H. Kim, Adv. Health Mater. 2015, 4, 1359; b) S. Hong, D. Sycks, H. F. Chan, S. Lin, G. P. Lopez, F. Guilak, K. W. Leong, X. Zhao, Adv. Mater. 2015, 27, 4035.

[42] a) T. Zehnder, B. Sarker, A. R. Boccaccini, R. Detsch, Biofabrication 2015, 7, 025001; b) M. Farokhi, F. Jonidi Shariatzadeh, A. Solouk, H. Mirzadeh, International Journal of Polymeric Materials and Polymeric Biomaterials 2019, 69, 230; c) A. C. Hernandez-Gonzalez, L. Tellez-Jurado, L. M. Rodriguez-Lorenzo, Carbohydr. Polym. 2020, 229, 115514.

[43] Q. Liu, G. Nian, C. Yang, S. Qu, Z. Suo, Nat. Commun. 2018, 9, 846. 
This work presents a new type of electroactive hydrogels capable of actuating outside water. The bending actuation is studied in different media: water, ionic liquid and air. A mechanism and a mathematical model to explain this behaviour are introduced. Finally, a gripper and a soft fingertip are presented as demonstrators, and 3D printing as a manufacturing method.

Keyword Hydrogel robotics

Antonio López-Díaz ${ }^{\dagger}$, Ana Martín-Pacheco ${ }^{\dagger}$, Antonio M. Rodríguez, M. Antonia Herrero, Andrés S. Vázquez*, Ester Vázquez*

Concentration Gradient-Based Soft Robotics: Hydrogels Out of Water

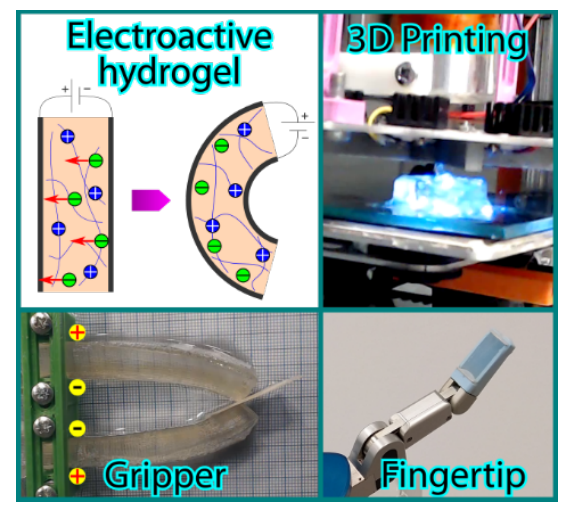


Copyright WILEY-VCH Verlag GmbH \& Co. KGaA, 69469 Weinheim, Germany, 2018.

\section{Supporting Information}

\section{Concentration Gradient-Based Soft Robotics: Hydrogels Out of Water}

Antonio López-Díaz ${ }^{\dagger}$,Ana Martín-Pacheco ${ }^{\dagger}$, Antonio M. Rodríguez, M. Antonia Herrero, Andrés S. Vázquez*, Ester Vázquez*

\section{Experimental section}

Photoinitiator synthesis: NaTPO (sodium (2,4,6-trimethylbenzoyl)phenylphosphinate) was prepared according to the procedure described by Noe et al. ${ }^{[1]}(2,4,6-$ Trimethylbenzoyl)phenylphosphine acid ethyl ester $(3.22 \mathrm{mmol})$ and sodium iodate (3.55 mmol) was dissolved in methyl ethyl ketone $(5 \mathrm{ml})$. The reaction was carried out in a flask with an adapted reflux condenser at $70^{\circ} \mathrm{C}$ for 24 hours.

The resulting precipitate was filtered off, washed with methyl ethyl ketone $(10 \mathrm{ml})$ and polyethylene $(2 \times 10 \mathrm{ml})$. The solid was dried under high vacuum and then recrystallized from diethyl ether to yield the product as a yellowish powder. ${ }^{1} \mathrm{H}$ NMR, ${ }^{13} \mathrm{C}$ NMR and ${ }^{31} \mathrm{P} \mathrm{NMR}$ spectra were recorded in methanol- $\mathrm{d}^{4}$ solutions at $298 \mathrm{~K}$ on a Bruker Avance 400 spectrometer ( $\delta$ in ppm, $\mathrm{J}$ in $\mathrm{Hz}$ ) at $1 \mathrm{H}$ operating frequency of $399.9 \mathrm{MHz} \cdot{ }^{1} \mathrm{H},{ }^{13} \mathrm{C}$ and ${ }^{31} \mathrm{P}$ spectra were referenced using the solvent signal as an internal standard:

- $\quad{ }^{1} \mathrm{H}$ NMR (400 MHz, Methanol-d $\left.\mathrm{d}^{4}\right) \delta 7.78(\mathrm{t}, \mathrm{J}=8 \mathrm{~Hz}, 2 \mathrm{H}), 7.43(\mathrm{t}, \mathrm{J}=8 \mathrm{~Hz}, 1 \mathrm{H}), 7.38$ $7.31(\mathrm{~m}, 2 \mathrm{H}), 6.74(\mathrm{~s}, 2 \mathrm{H}), 2.22(\mathrm{~s}, 3 \mathrm{H}), 2.09(\mathrm{~s}, 6 \mathrm{H})$.

- ${ }^{13} \mathrm{C}$ NMR (101 MHz, Methanol-d $\left.{ }^{4}\right) \delta 141.13,140.73,139.43,137.39,136.13,134.99$, $133.96,133.87,131.96,131.93,129.02,128.74,128.62,21.13,19.77$.

- ${ }^{31} \mathrm{P}$ NMR (162 MHz, Methanol-d $\left.{ }^{4}\right) \delta 10.83$. 
Preparation and characterization of FLG: The preparation of FLG aqueous dispersions was carried out by a ball-milling methodology. ${ }^{[2]}$

In a typical experiment, graphite $(7.5 \mathrm{mg})$ and melamine $(22.5 \mathrm{mg})$ were ball-milled in a stainless-steel flask that contained ten stainless-steel balls $(1 \mathrm{~cm}$ diameter) using a Retsch PM 100 ball mill. The resulting solid mixture was transferred to an Erlenmeyer flask using $20 \mathrm{ml}$ of deionized water and ultrasonication, which led to a dark suspension. Melamine was removed by dialysis against hot deionized water $\left(70^{\circ} \mathrm{C}\right)$ thought a dialysis membrane (MWCO: $6-8$ $\mathrm{kDa})$. During the dialysis, the permeate was replaced every 2 hours at least five times and overnight to achieve the purification. Graphite was removed by allowing the mixture to settle for five days. The resulting supernatant, which was stable in water, was carefully extracted by pipetting to provide FLG water dispersions. The corresponding FLG powder was obtained by freeze-drying.

TA Instruments Q50 thermogravimetric analysis (TGA) equipment was used for thermal analysis. The method consisted of a $100{ }^{\circ} \mathrm{C}$ isothermal step for $20 \mathrm{~min}$, followed by an increase at $10{ }^{\circ} \mathrm{C}$ per minute up to $800{ }^{\circ} \mathrm{C}$ in a nitrogen atmosphere. TGA (Figure S1a) verified the low concentration of oxygen groups in the as-prepared FLG. A weight loss of $9 \%$ was observed at $600{ }^{\circ} \mathrm{C}$.

Raman spectra were recorded on an Invia Renishaw microspectrometer equipped with a 532 $\mathrm{nm}$ point-based laser. Raman samples were prepared by dropping dilute aqueous FLG dispersions onto silicon oxide surfaces (WRS Materials). The spectra were treated for baseline correction, noise filtering and normalization. Moreover, the spectra were fitted with Lorentzianshaped bands to ascertain band positions, widths and intensities. The Raman spectrum (Figure S1b) displays the two most intense peaks of graphene: G and 2D peaks, at around 1580 and $2700 \mathrm{~cm}^{-1}$, respectively. The $\mathrm{G}$ peak is typical of carbon structures dominated by $\mathrm{sp}^{2}$ domains, while the 2D peak reveals the number of layers. Moreover, the D peak, which indicates a disordered graphene structure ( $\mathrm{sp}^{3}$ domains), and the $\mathrm{D}^{\prime}$ peak appear at around 1345 and 1600 
$\mathrm{cm}^{-1}$, respectively. Additional useful information can be obtained from the full width at half maximum of the 2D peak (FWHM(2D)) and the ratio between the 2D and the G peak intensities $\left(\mathrm{I}_{2 \mathrm{D}} / \mathrm{I}_{\mathrm{G}}\right)$, which are $60.6 \mathrm{~cm}^{-1}$ and $0.45 \mathrm{~cm}^{-1}$, respectively, confirming that the obtained sample is few-layer graphene. Furthermore, the ratio between the $\mathrm{D}$ and the $\mathrm{G}$ peak intensities $\left(\mathrm{I}_{\mathrm{D}} / \mathrm{I}_{\mathrm{G}}\right)$ is 0.4 , which is attributed to the defects at the edges of the sub-micrometer flakes.

The low number of defects was corroborated by the elemental analysis shown in Figure S1c. Transmission Electron Microscopy (TEM) was carried out on a Jeol 2100 HRTEM system. The samples were prepared by immersion of the nickel grid (LC200-Ni Lacey Carbon Grid from Electron Microscopy Sciences) into the dilute FLG dispersions. The samples were dried under vacuum prior to analysis. A representative TEM image is shown in Figure S1d.

Electroresistivity measurements were performed by the standard four-probe technique using a Multi-Height Microposition Probe with an RM3000 Unit (Bridge Technologies USA) at room temperature. The conductivity was determined on the solid residue from a known volume of nanomaterial dispersion. For this, $10 \mathrm{ml}$ of $0.1 \mathrm{mg} \cdot \mathrm{ml}^{-1}$ of the nanomaterial dispersion was filtered in a tared membrane, dried under vacuum for two hours and weighed.

Hydrogels characterization: The swelling degree is defined by $S D=\frac{w-w_{d r y}}{w_{d r y}}$, where $w$ is the weight of a swollen hydrogel sample, and $w_{d r y}$ is the weight of the same sample in the dry state, also called a xerogel. This parameter was measured as follows: $\mathrm{CN}$ and $\mathrm{CNG}$ xerogels were immersed in excess deionized water at room temperature, and their weights were determined at regular intervals until a constant weight was achieved, which was the maximum swelling degree. Prior to weighing, the excess water on the hydrogel surfaces was removed with a piece of filter paper. The measurement was repeated at least three times in order to obtain an average maximum swelling degree and the corresponding standard deviation.

The swelling degree is closely related to the mass percentage of water, as stated below: 


$$
S D=\frac{w-w_{d r y}}{w_{d r y}}=\frac{\left(w_{d r y}+w_{w a t e r}\right)-w_{d r y}}{w_{d r y}}=\frac{w_{\text {water }}}{w_{d r y}}
$$

$$
\begin{gathered}
\text { water } \%=\frac{w_{\text {water }}}{w} 100 \%=\frac{w_{\text {water }}}{w_{\text {dry }}+w_{\text {water }}} 100 \%=\frac{\frac{w_{\text {water }}}{w_{\text {dry }}}}{\frac{w_{\text {dry }}}{w_{\text {dry }}}+\frac{w_{\text {water }}}{w_{\text {dry }}}} 100 \%= \\
=\frac{S D}{1+S D} 100 \%
\end{gathered}
$$

Compressive tests were performed on hydrogels at room temperature with a testing machine (Mecmesin Multitest 2.5-i). Cylindrical-shaped hydrogel samples were prepared, dried at $50{ }^{\circ} \mathrm{C}$ and swollen again at swelling degrees of 1, 5, 10, 25 (6 hydrogels each swelling degree). These hydrogels were uniaxially compressed between two plates at a rate of $6 \mathrm{~mm} \cdot \mathrm{min}^{-1}$, using a cell load of $50 \mathrm{~N}$, until the $50 \%$ of the initial height of each hydrogel was reached. The forcedeformation curve was converted into a stress-strain curve, given the dimensions of each sample. Young's Modulus was calculated in the linear range of the stress-strain curve (between $2 \%$ and $10 \%$ of strain, in this case).

Cryo-SEM analysis of CN and CNG was carried out on a GeminiSEM 500 from Zeiss coupled with a Quorum cryo-SEM. The CN and CNG hydrogel samples were prepared as follows. Firstly, the samples were swollen until the maximum swelling degree was reached. The gels were then frozen in $\mathrm{N}_{2}$, and the slush was manually fractured. The condensed surface water was sublimed by controlling the temperature at $-90{ }^{\circ} \mathrm{C}$ for $8 \mathrm{~min}$. The resulting samples were sputtered with gold (45 s), and finally, they were introduced into the microscope sample stage for examination at an accelerating voltage of $5 \mathrm{kV}$.

Measurement of the bending behaviour: A home-made system based on a Petri dish with a 3D printed polylactic acid (PLA) was used. This system held the electrodes and housed the liquid 
medium in which the tests were performed. Platinum-based electrodes $(30 \mathrm{~mm}$ long, $10 \mathrm{~mm}$ wide, $0.55 \mathrm{~mm}$ thick) were used, and they were adjusted to this system and separated by $40 \mathrm{~mm}$. A current sensor (ACS712-5A) and a camera (Logitech C922) were adapted to this system to record the bending effect.

The study was carried out using hydrogel samples with $23.5 \pm 0.8,5.8 \pm 1.0$ and $2.9 \pm 0.2 \mathrm{~mm}$ length, width and thickness, respectively.

The results from the bending behaviour study were obtained as follows. Once the electric field was applied, the camera recorded the hydrogel and the computer read data from the Arduino board and the current sensor (Figure S2). After the test, the video was processed to obtain the curvature of the hydrogel at different times. The curvature $(K)$ was defined as the inverse of the radius $(R)$, which is shown in Figure $2 \mathrm{a}$. The sign criteria for plotting the curvature was as follows: in an aqueous medium, the curvature towards the anode is considered positive, while in ionic liquid and ambient-air conditions, the curvature towards the cathode is considered positive.

To study the bending effect of the hydrogel in ambient-air conditions using flexible electrodes, the as-prepared $\mathrm{CN}$ and $\mathrm{CNG}$ were swollen at $S D 25$ with deionized water, cut $(\sim 80 \times 10 \times 15$ $\mathrm{mm}$ ) and then frozen with liquid $\mathrm{N}_{2}$. Once frozen, the platinum paper-based electrodes were attached to both parts of the hydrogels. The hydrogels were left in ambient conditions until thawing prior to the study. The bending behaviour was studied on a glass-based surface using vaseline oil to reduce the friction between the glass surface and the hydrogel.

Ionic liquid synthesis: 1-Butyl-3-methylimidazolium tetrafluoroborate (BMIMBF 4 ) ionic liquid was synthesized as described by Dharaskar et al. ${ }^{[3]}$

1-Butyl-3-methylimidazolium bromide was prepared as follows: bromobutane (0.8 mol) and 1methyimidizole $(0.1 \mathrm{~mol})$ were mixed in a round-bottomed flask fitted with a reflux condenser. The mixture was stirred for $48 \mathrm{~h}$ at $70{ }^{\circ} \mathrm{C}$. The product was separated by decantation using ethyl 
acetate as the solvent, and the bromide derivative was obtained by removing the solvent on a rotary evaporator.

The counter-anion was changed from bromide to tetrafluoroborate by mixing the as-prepared 1-butyl-3-methylimidazolium bromide $(0.03 \mathrm{~mol})$ and sodium tetrafluoroborate $(0.03 \mathrm{~mol})$ using acetone as solvent. The mixture was vigorously stirred for $10 \mathrm{~h}$ at $40{ }^{\circ} \mathrm{C}$. The reaction mixture was then filtered using a $0.2 \mu \mathrm{m}$ pore Millipore filter and dichloromethane as the solvent to separate the 1-butyl-3-methylimidazolium tetrafluoroborate from the sodium bromide by-product. Finally, the ionic liquid was obtained by removing the solvent on a rotary evaporator.

${ }^{1} \mathrm{H}$ NMR and ${ }^{13} \mathrm{C}$ NMR spectra were recorded in methanol- $\mathrm{d}^{4}$ solutions at $298 \mathrm{~K}$ on a Bruker Avance 400 spectrometer ( $\delta$ in ppm, J in $\mathrm{Hz}$ ) at $1 \mathrm{H}$ operating frequency of $399.9 \mathrm{MHz} .{ }^{1} \mathrm{H}$ and ${ }^{13} \mathrm{C}$ spectra were referenced using the solvent signal as an internal standard:

- $\quad{ }^{1} \mathrm{H}$ NMR (400 MHz, Methanol-d $\left.\mathrm{d}^{4}\right) \delta 8.85(\mathrm{~s}, 1 \mathrm{H}), 7.7$ (d, J = 1.6 Hz, 1H), 7.6 (d, J = 1.6 $\mathrm{Hz}, 1 \mathrm{H}), 4.26(\mathrm{t}, \mathrm{J}=7.3 \mathrm{~Hz}, 2 \mathrm{H}), 3.97(\mathrm{~s}, \mathrm{~J}=2.6 \mathrm{~Hz}, 3 \mathrm{H}), 1.89(\mathrm{~m}(5), \mathrm{J}=7.5 \mathrm{~Hz}, 2 \mathrm{H})$, $1.37(\mathrm{~m}(6), \mathrm{J}=7.4 \mathrm{~Hz}, 2 \mathrm{H}), 0.95(\mathrm{t}, \mathrm{J}=7.4 \mathrm{~Hz}, 3 \mathrm{H})$.

- ${ }^{13} \mathrm{C}$ NMR (101 MHz, Methanol-d $\left.{ }^{4}\right) \delta 137.50,124.58,123.32,50.25,36.44,32.76,20.08$, 13.67.

Stiffness and volume change: A home-made system based on a Petri dish with a 3D printed polylactic acid (PLA) was used. This system mimicked the phalanx and housed the electrodes and the hydrogel. Platinum-based electrodes (30 $\mathrm{mm}$ long, $10 \mathrm{~mm}$ wide, $0.55 \mathrm{~mm}$ thick) were used, and they were adjusted to this system and separated by $50 \mathrm{~mm}$.

The testing machine (Mecmesin Multitest 2.5-i) used to apply the mechanical force was adapted to the hydrogel dimensions with a 3D printed PLA piece.

The study was carried out on hydrogel samples that were $49.6 \pm 1.3 \mathrm{~mm}$ long, $18.0 \pm 0.3 \mathrm{~mm}$ wide and $8.7 \pm 0.4 \mathrm{~mm}$ high. 
The volume change in a particular region of the hydrogel was obtained by compressing the hydrogel by $2 \mathrm{~mm}$ while maintaining this penetration constant over time. An electric field was applied, and the mechanical force exerted by the hydrogel was measured. Note that the electric field was kept constant at $20 \mathrm{~V}$ or inverted (from $20 \mathrm{~V}$ to $-20 \mathrm{~V}$ ) depending on the experiment. The changes in stiffness were evaluated by compressing a region of the hydrogel periodically up to $0.2 \mathrm{~N}$ on the testing machine. The displacement was measured while applying an electric field at $20 \mathrm{~V}$.

Temperature changes: The temperature changes in the hydrogel were measured while applying an electric field. The samples and the electrodes (separated $35 \mathrm{~mm}$ ) were held by a 3D printed PLA part. The temperature was measured during the experiment using an infrared thermal camera (Flir A65).

The hydrogels were swollen at $S D 9.3$ with 0.05 м aqueous LiCl. The voltage applied was 14 V for 7 minutes approximately.

The camera registered the temperature of the $\mathrm{CN}$ and $\mathrm{CNG}$ hydrogels during this time. Temperature values from the thermal images were extracted at points P1, P2 and P3 (Figure S9).

$3 D$ printing and inks: Rheological studies of neat CN-ALG inks were carried out on a DHR-1 Rheometer (TA Instruments) using a $40 \mathrm{~mm}$ diameter stainless steel parallel plate and a Peltier system, working in oscillatory shear and flow mode. For all measurements, the gap between the geometry and the Peltier system was $1000 \mu \mathrm{m}$. The temperature was kept constant at $25^{\circ} \mathrm{C}$. 3D printing was performed with a 3Drag v1.2 printing system (replica of the Velleman K8200 printer) modified for use with our hydrogel inks. This printer comprises a printing bed that moves in the $\mathrm{X}$ and $\mathrm{Y}$ axes and a syringe injector, which contained the hydrogel printing solution, mounted in the Z-axis. The printer was adapted with a UV light system formed by 
four LEDs operating at $365 \mathrm{~nm}$ (1200 mW of power each) (Figure 8b). The software RepetierHost V2.0.1. was used to manipulate and control the 3D printer. 3D printing models were designed (SolidWorks) and sliced into layers, using the software Repetier-Host and CuraEngine as the slicer engine $(0.5-0.7 \mathrm{~mm}$ slicing thickness, with $0.5 \mathrm{~mm}$ being the best choice).

Square scaffolds ( $25 \times 25 \mathrm{~mm}$, up to 25 layers) were selected as the preferred model to compare all of the printed samples. Samples were printed with a $0.50 \mathrm{~mm}$ (inner diameter) syringe tip while several parameters were varied, including filling (40-100\%), layer thickness $(0.5-0.7$ $\mathrm{mm})$ and printing speed $\left(20-60 \mathrm{~mm} \cdot \mathrm{s}^{-1}\right)$.

\section{Mathematical model}

The Nernst-Planck equation (Equation S2) describes the movement of a certain species $i$ in a medium:

$\vec{J}_{i}=-D_{i}\left(\vec{\nabla} c_{i}+z_{i} c_{i} f \vec{\nabla} \phi\right)$

where $\vec{J}_{i}$ is the flux of the species $i$ in the medium, $D_{i}$ is its diffusion coefficient, $c_{i}$ is its concentration (spatial-dependent), $z_{i}$ is its valence, $\phi$ is the electric potential and $f=F / R T$ is a constant coefficient defined by the Faraday constant $(F)$, the gas constant $(R)$ and the temperature $(T)$.

If there is no generation or consumption of the species $i$, Equation $\mathrm{S} 3$ is obtained performing a mass balance:

$\frac{\partial c_{i}}{\partial t}=\vec{\nabla}\left[D_{i}\left(\vec{\nabla} c_{i}+z_{i} c_{i} f \vec{\nabla} \phi\right)\right]$ 
Since the electric field is applied in a particular direction $(y)$ in the case presented here, Equation $\mathrm{S} 3$ is simplified into a one-dimensional partial differential equation (PDE):

$\frac{\partial c_{i}}{\partial t}=\frac{\partial}{\partial y}\left(D_{i} \frac{\partial c_{i}}{\partial y}+D_{i} z_{i} c_{i} f \frac{\partial \phi}{\partial y}\right)$

This PDE can be solved (i.e., to obtain $\left.c_{i} \equiv c_{i}(y, t)\right)$ with the appropriate initial and boundary conditions, which reflect some features or considerations of our hydrogel case:

- Initial condition: the initial concentration of species $i$ is homogeneous (constant and position independent).

$c_{i}(y, t=0)=C_{i 0}$

- Boundary conditions: there is no flux of the species $i$ (i.e., mobile ions) on the borders ( $y=0$ and $y=h$, the planes where the electrodes are placed). This condition means that the total quantity of water inside the hydrogel remains constant since the solvated ions do not flow out the hydrogel given the hydrophilic nature of the hydrogel.

$\left.D_{i}\left(\frac{\partial c_{i}}{\partial y}+z_{i} c_{i} f \frac{\partial \phi}{\partial y}\right)\right|_{y=0, h}=0$

- The electric potential is considered linear, neglecting the effect that mobile ions can cause it.

$\phi(y, t)=V \frac{y}{h}$ 
Given these conditions (Equation S5, S6 and S7), the PDE (Equation S4) can be solved once for each of the species of mobile ions presented in the hydrogel. Knowing the concentrations of these species, the water concentration distribution inside the hydrogel $(C W(y, t))$ can be estimated:

$$
C W(y, t)=\frac{\alpha_{f} C_{f 0}+\sum_{i} \alpha_{i} c_{i}(y, t)}{\alpha_{f} C_{f 0}+\sum_{i} \alpha_{i} C_{i 0}} C W_{0}
$$

where $c_{i}(y, t)$ are the solutions of Equation $\mathrm{S} 4$ for each species. $C W_{0}$ is the initial homogeneous concentration of water in the hydrogel and $C_{f 0}$ represents the uniform concentration of charges bound to the polymer network (constant value in time), while $C_{i 0}$ is the initial uniform concentration of each species. The coefficients $\alpha_{i}$ represent the percentage of water solvated in each kind of mobile ion, while $\alpha_{f}$ represents the percentage of water anchored to the network. Note that $\alpha_{f}+\sum_{i} \alpha_{i}=1$ and $\frac{1}{h} \int_{0}^{h} C W(y, t) d y=C W_{0}$, which is consistent with the assumption that the total quantity of water inside the hydrogel remains constant.

The model described above estimates the water concentration distribution with respect to time $(C W(y, t))$, which will be used to perform mechanical calculations in order to predict the curvature of a hydrogel strip.

These mechanical calculations are based on the Timoshenko model, ${ }^{[4]}$ which has the following expression for the curvature $(K)$ :

$$
K=\frac{1}{R}=\frac{\delta_{2}-\delta_{1}}{\frac{h}{2}+\frac{2\left(E_{1} I_{1}+E_{2} I_{2}\right)}{h}\left(\frac{1}{E_{1} a_{1}}+\frac{1}{E_{2} a_{2}}\right)}
$$

where subindexes denote the layer, $h$ is the total height of the whole strip, $a_{j}$ is the height of each layer, $E_{j}$ is the Young's Modulus, $I_{j}$ is the planar moment of inertia and $\delta_{j}$ is: 
$\delta_{j} \approx \frac{1}{3} \frac{\Delta V_{j}}{V_{j 0}}, \quad j=1,2$

in which $\Delta V_{j}$ is the volume change of layer $j$ and $V_{j 0}$ its initial volume.

In the bi-metal case studied by Timoshenko, the layers are directly defined, but in the hydrogel, this is not straightforward. The criterion used to divide the hydrogel beam into two layers is the neutral axis, which is defined by the plane in which there are no longitudinal stresses (Figure 5a). We assume that the internal stress is proportional to the water concentration distribution, $\sigma \propto\left(C W(y, t)-C W_{0}\right)$, so this neutral axis corresponds to:

$y^{*}: C W\left(y^{*}, t\right)=C W_{0}$

With this definition, the following expressions are valid:

$$
\begin{aligned}
& a_{1}=y^{*} \\
& a_{2}=h-y^{*} \\
& V_{1,0}=w L a_{1} \\
& V_{2,0}=w L a_{2} \\
& \Delta V_{1}(t)=w L \int_{0}^{a_{1}}\left(C W(y, t)-C W_{0}\right) d y \\
& \Delta V_{2}(t)=w L \int_{a_{1}}^{h}\left(C W(y, t)-C W_{0}\right) d y
\end{aligned}
$$

These expressions can be substituted into Equation S9 to obtain a value for the curvature with respect to time $(K(t))$, which is the finality of the model. 
a)

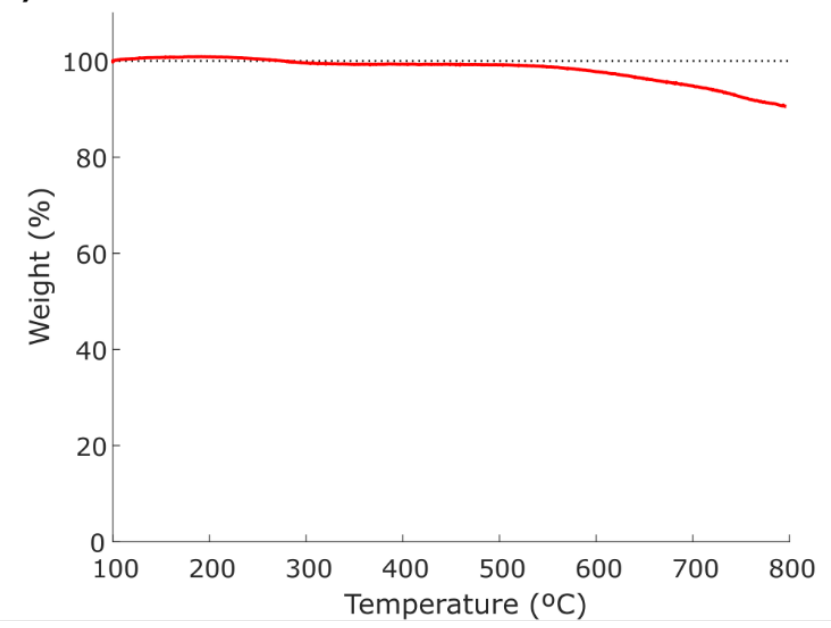

c)

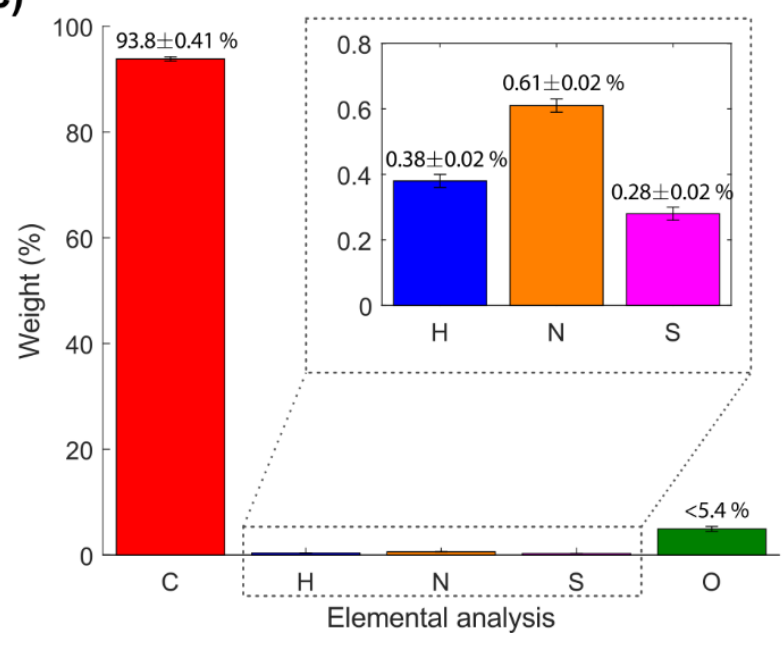

b)

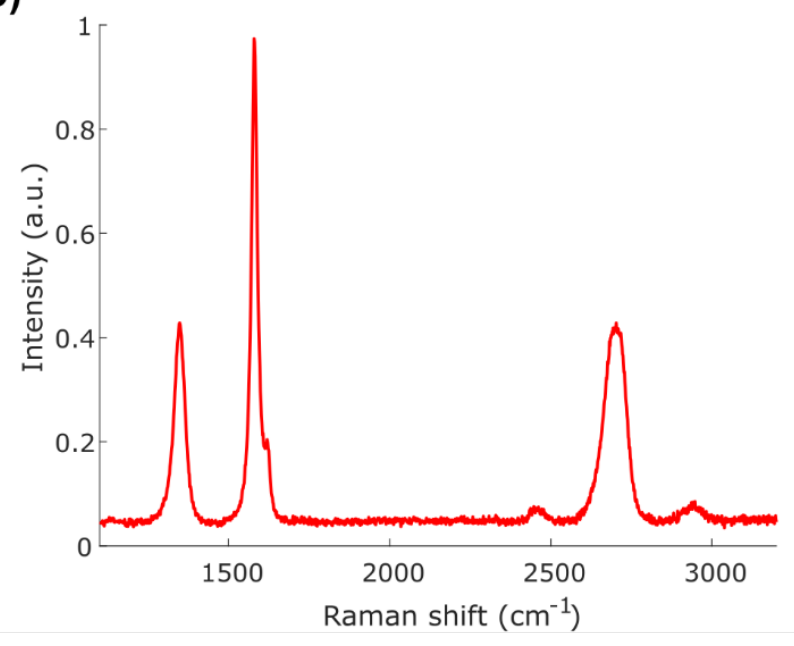

d)

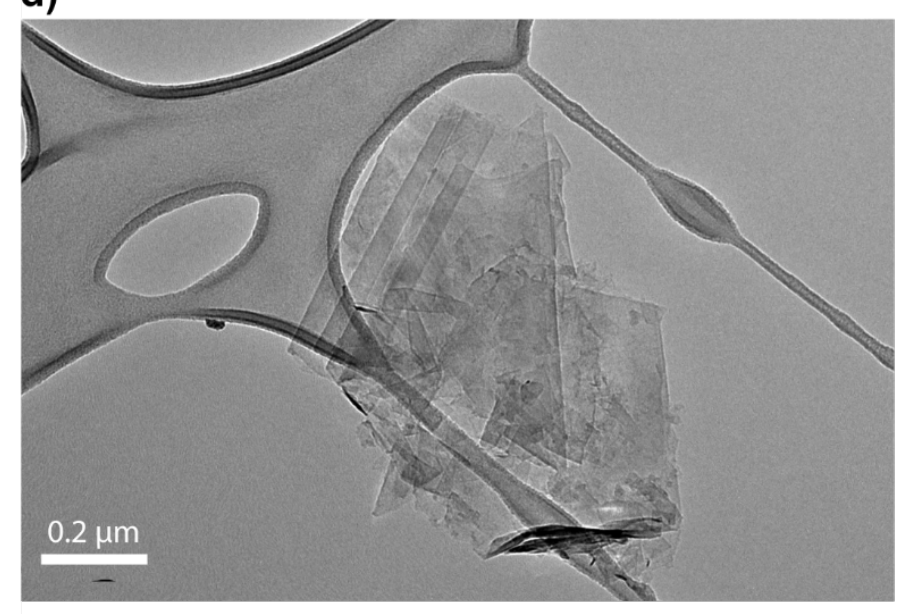

Figure S1. FLG characterization. (a) Thermogravimetric analysis. (b) Raman spectra. (c) Elemental analysis. (d) Transmission Electron Microscopy (TEM) image. 

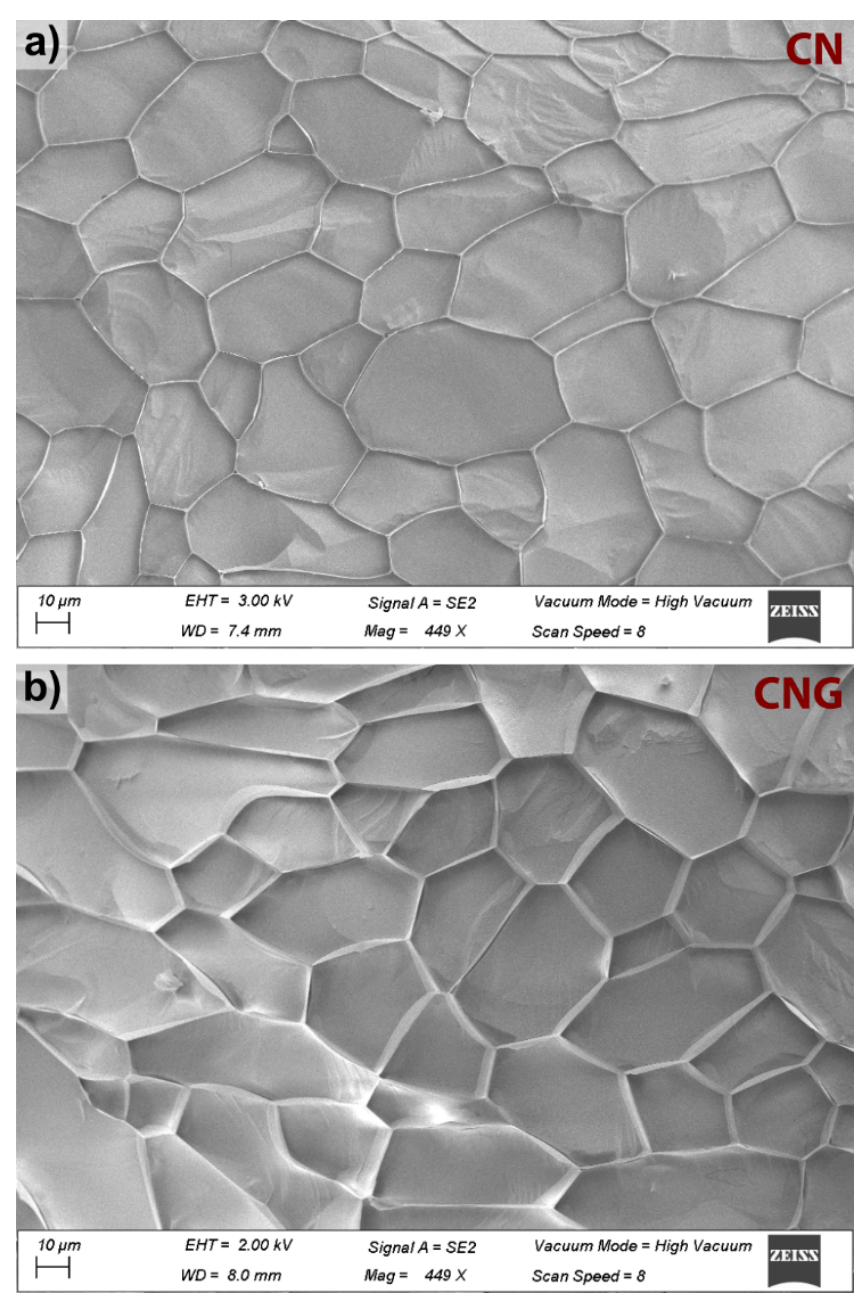

Figure S2. Cryo-SEM images. (a) CN. (b) CNG. 
a)
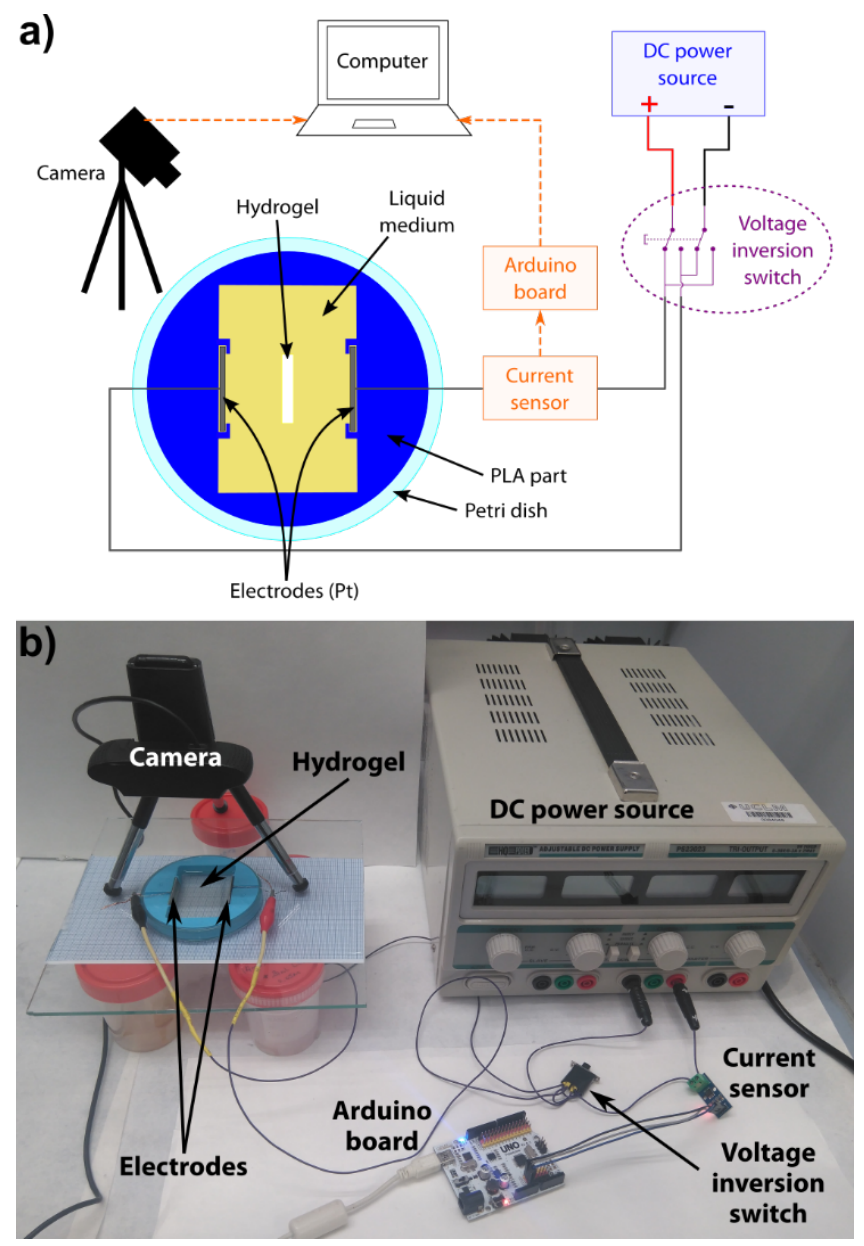

Figure S3. Setup to perform the bending tests. (a) Schematic diagram of the setup. (b) Assembled setup.

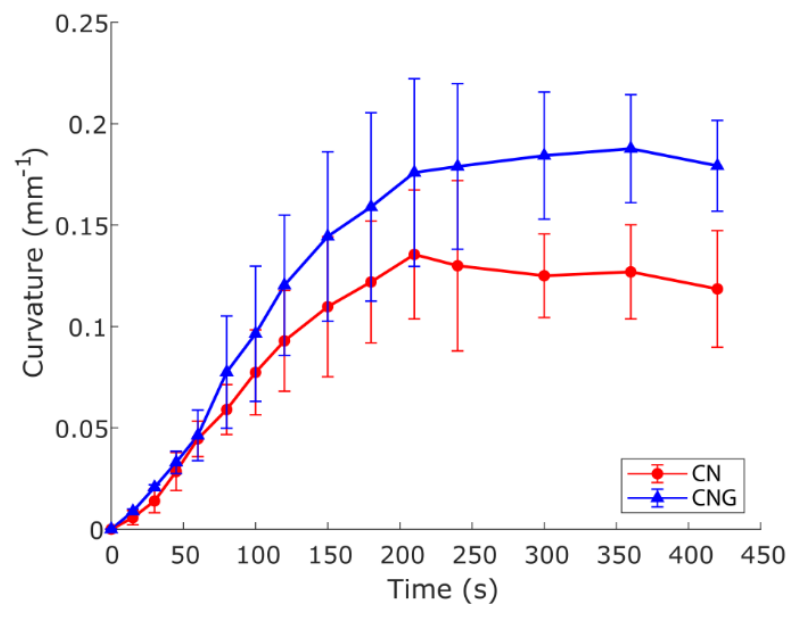

Figure S4. Constant voltage test in aqueous-based medium for $\mathrm{CN}$ and $\mathrm{CNG}$ hydrogel at $S D$ 2. 

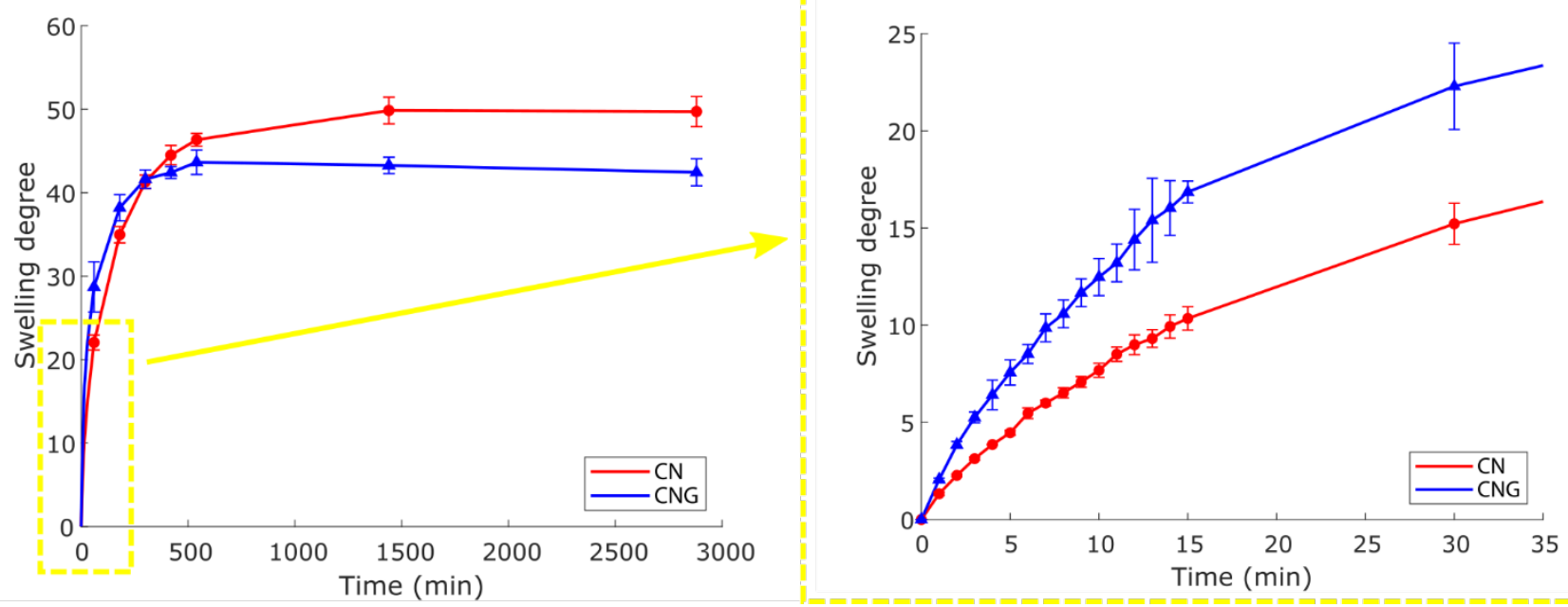

Figure S5. Swelling degree study for $\mathrm{CN}$ and $\mathrm{CNG}$ hydrogels in $0.05 \mathrm{~m}$ aqueous $\mathrm{LiCl}$ solution. 
a)

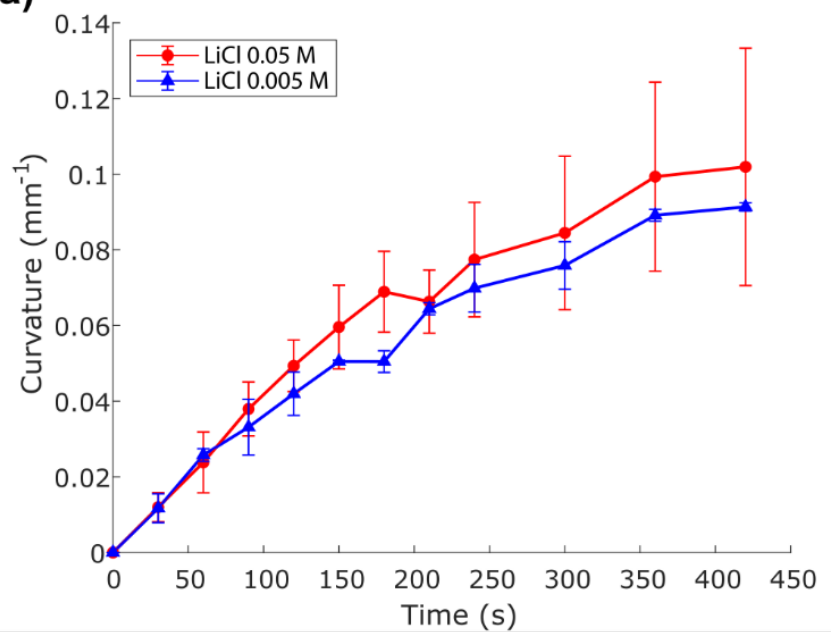

c)

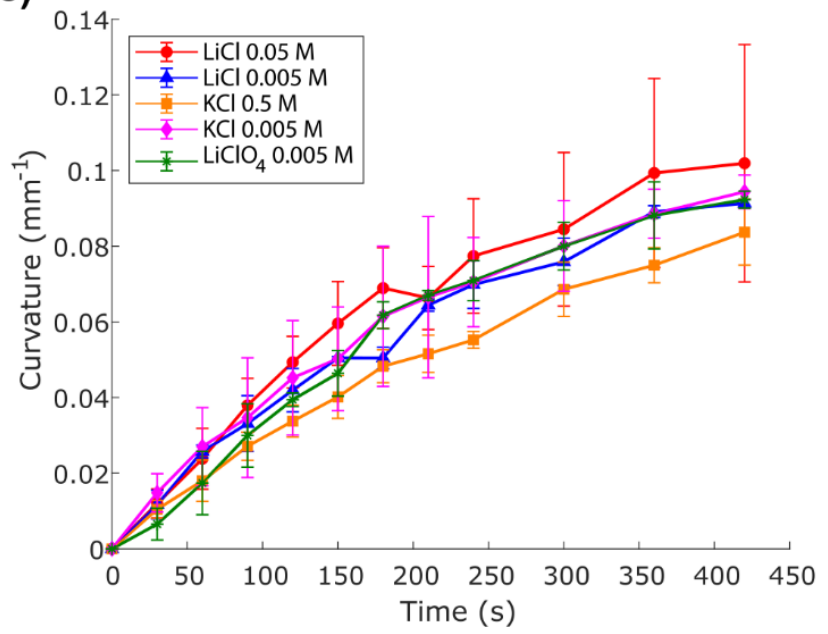

b)

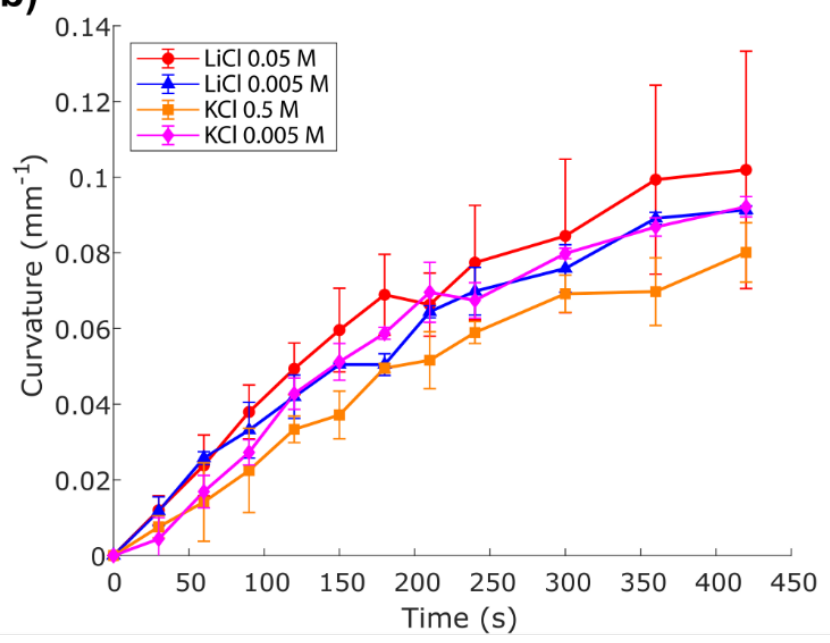

d)

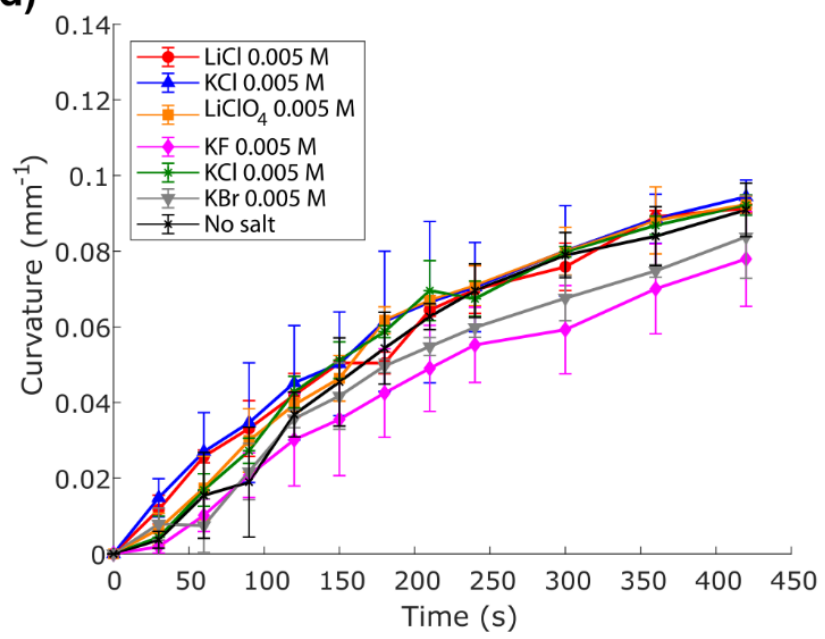

Figure S6. Bending of CN hydrogel swollen with different salts in ionic liquid medium. Constant voltage test in ionic liquid medium for: (a) $\mathrm{CN}$ hydrogel swollen with different $\mathrm{LiCl}$ concentrations. (b) $\mathrm{CN}$ hydrogel swollen with different salts on varying the cation. (c) $\mathrm{CN}$ hydrogel swollen with different salts on varying the anion. (d) CN hydrogel swollen with different salts and without salt. In all cases, the $S D$ is 25 .
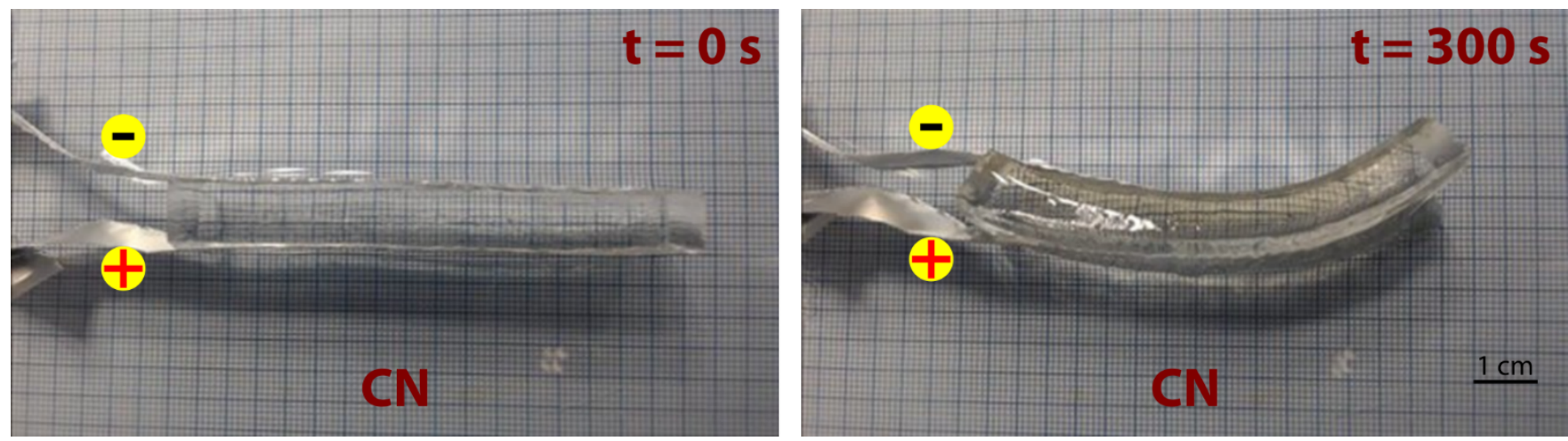

Figure S7. Curvature of $\mathrm{CN}$ under ambient-air conditions. 

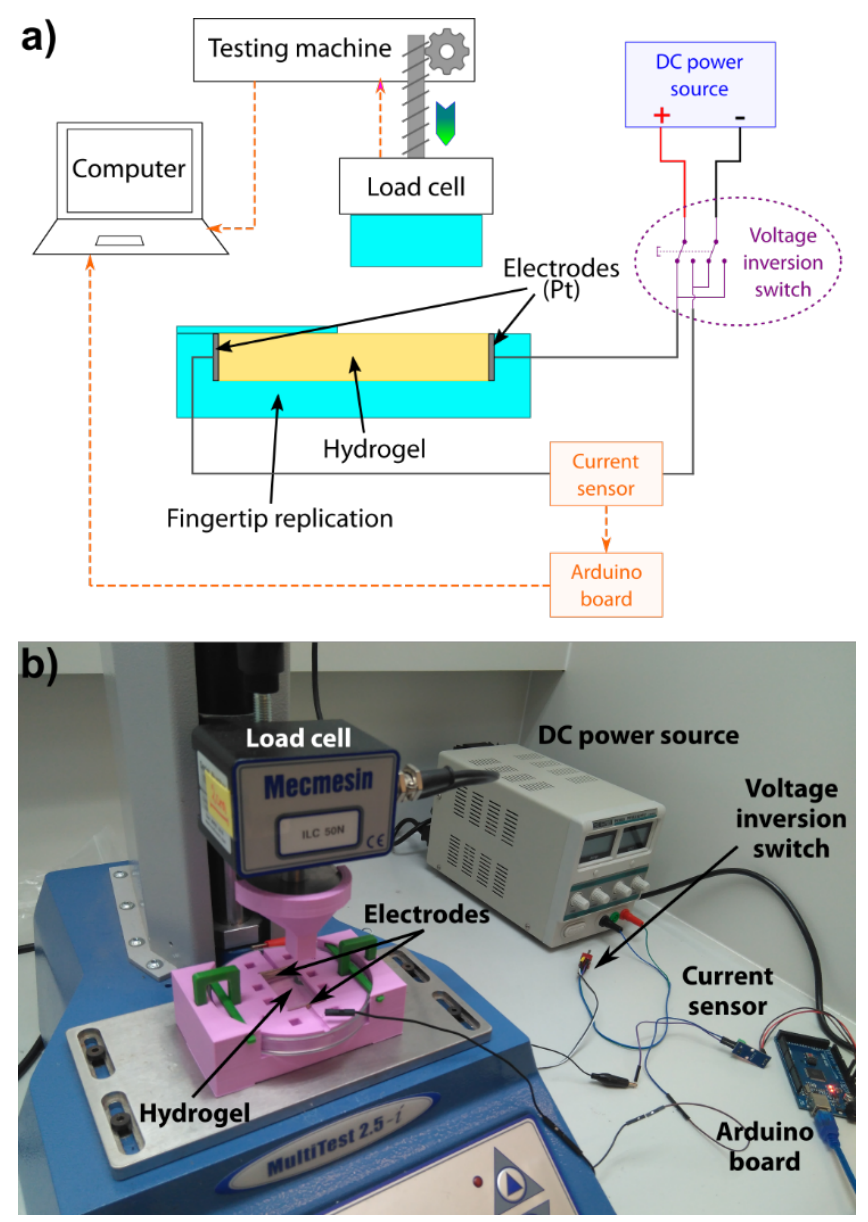

Figure S8. Setup to perform the fingertip tests. (a) Schematic diagram of the setup. (b) Assembled setup. 


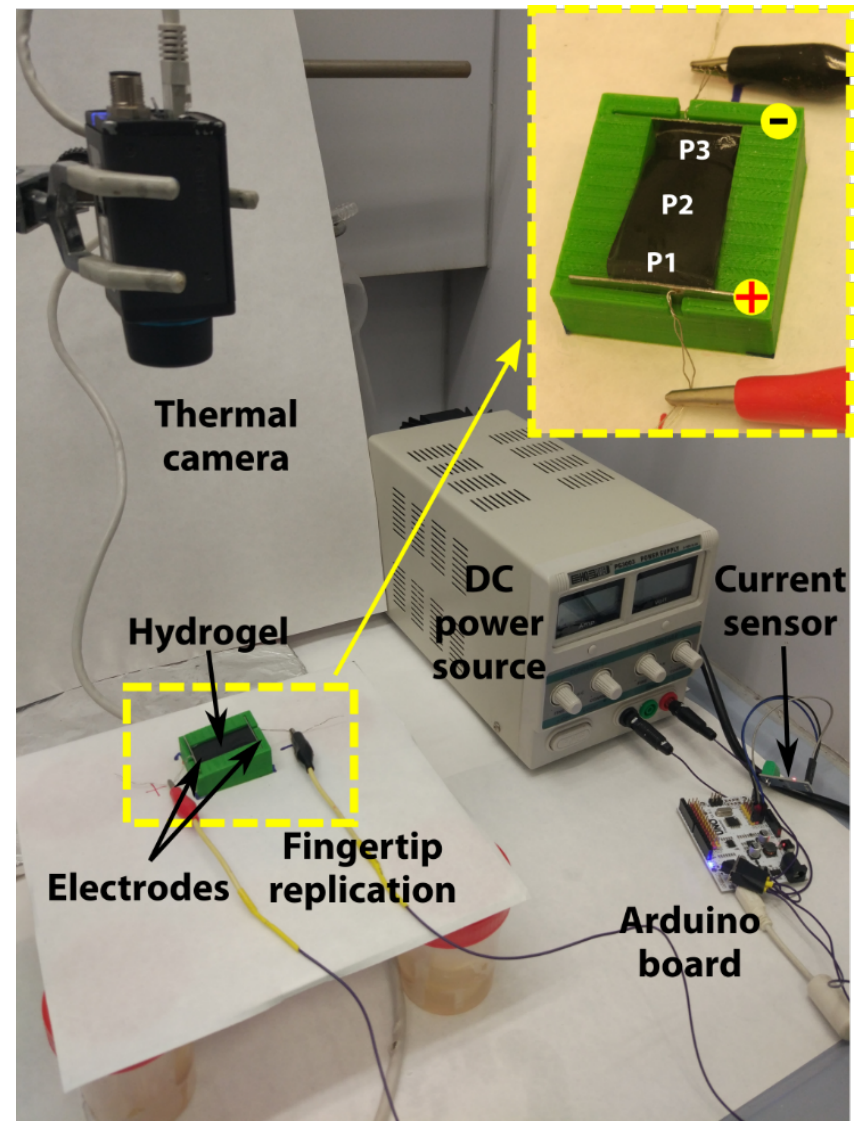

Figure S9. Setup to perform the temperature study on the fingertips and points considered for the study.

a)

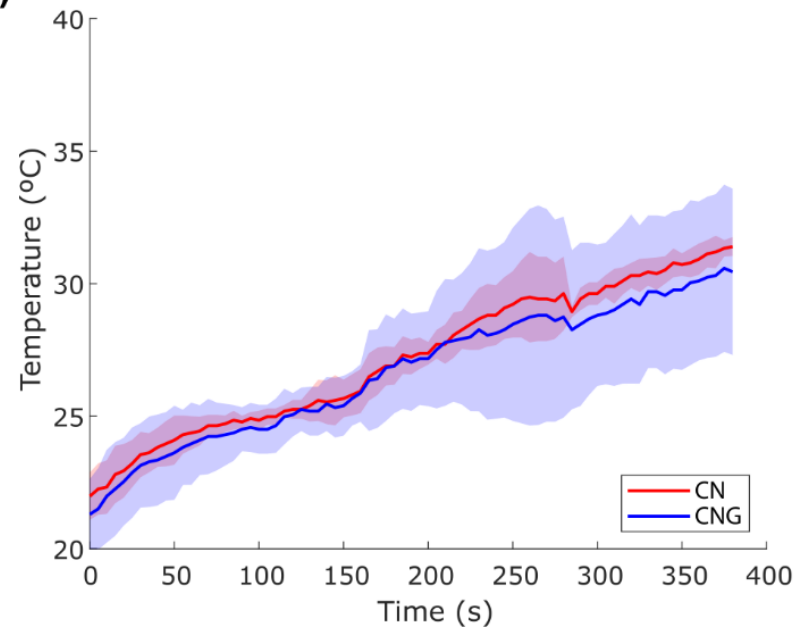

b)

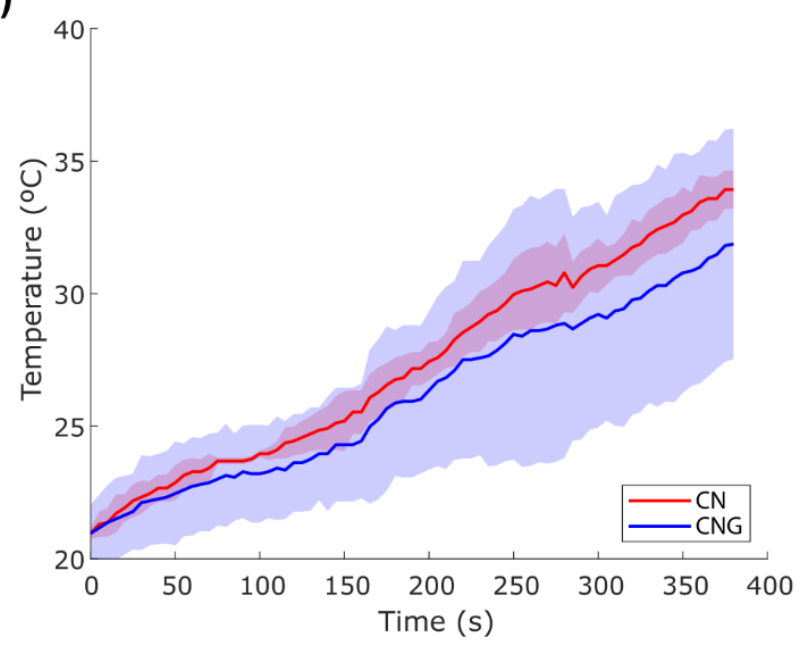

Figure S10. Results for the temperature at points $\mathrm{P} 1$ and $\mathrm{P} 2$. Evolution of the temperature of: (a) Point P1 in CN and CNG fingertips. (b) Point P2 in CN and CNG fingertips. 

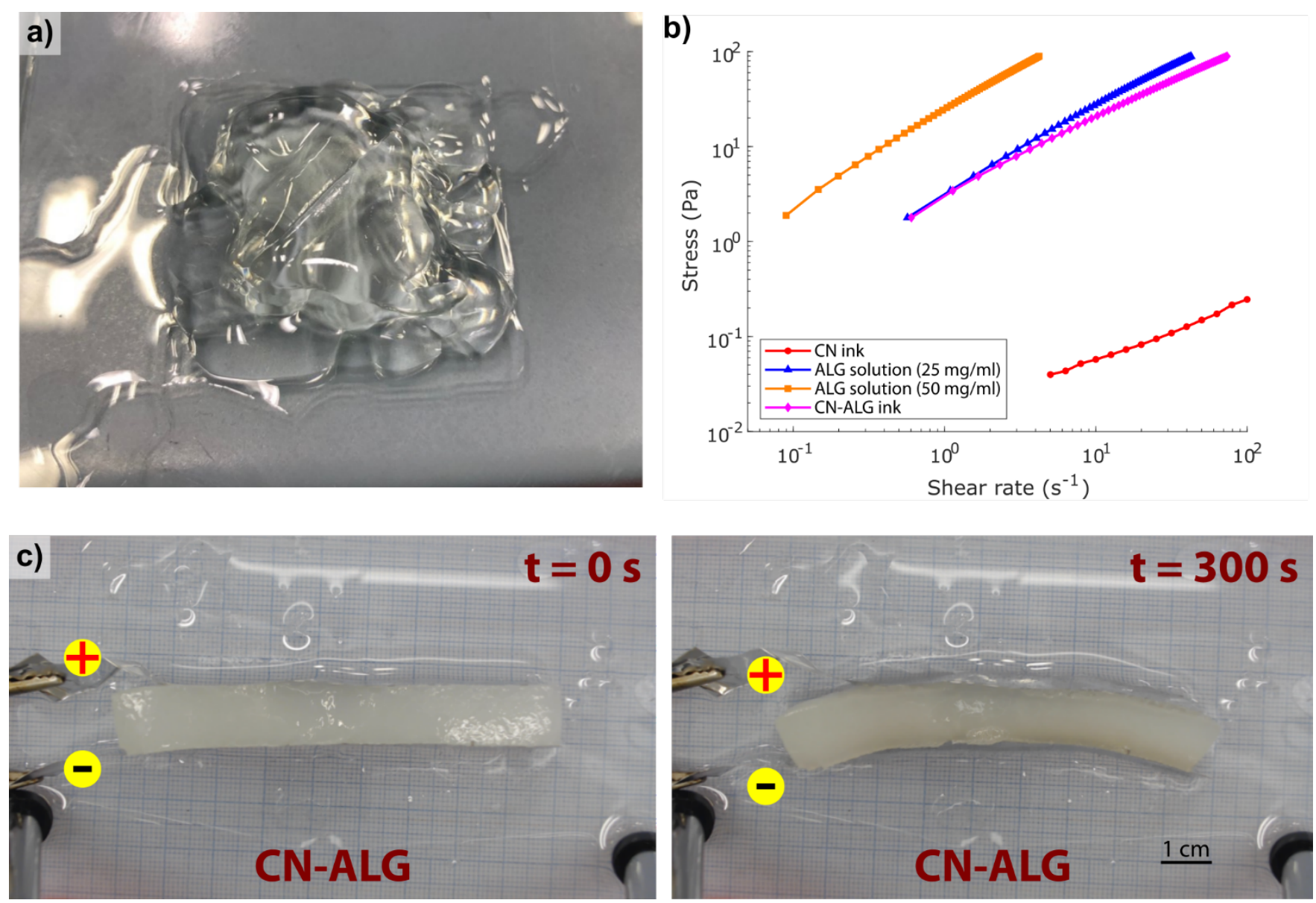

Figure S11. Pristine CN print, rheological properties of different inks and bending behaviour of CN-ALG. (a) Early attempt at 3D printing with the precursor of pristine CN hydrogels. (b) Zero-shear viscosity of different concentration of sodium alginate solution and hydrogel prepolymerized inks. (c) Curvature of CN-ALG at ambient-air conditions. 
Table S1. Parameter values used to run the model simulation for CN and VBS hydrogel.

\begin{tabular}{|c|c|c|c|}
\hline Parameter & Symbol & Value (CN) & Value (VBS) \\
\hline Voltage applied & $V$ & $4 \mathrm{~V}$ & $4 \mathrm{~V}$ \\
\hline Length & $L$ & $8.0 \cdot 10^{-2} \mathrm{~m}$ & $8.0 \cdot 10^{-2} \mathrm{~m}$ \\
\hline Height & $h$ & $1.0 \cdot 10^{-2} \mathrm{~m}$ & $1.0 \cdot 10^{-2} \mathrm{~m}$ \\
\hline Width & $w$ & $1.5 \cdot 10^{-2} \mathrm{~m}$ & $1.5 \cdot 10^{-2} \mathrm{~m}$ \\
\hline a)Young's Modulus & $E$ & $1.355 \cdot 10^{5} \cdot S D^{-0.99}+1.07 \cdot 10^{4}[\mathrm{~Pa}]$ & * \\
\hline Temperature & $T$ & $293 \mathrm{~K}$ & $293 \mathrm{~K}$ \\
\hline 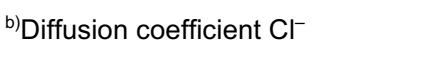 & $D_{C l}$ & $1.0 \cdot 10^{-9} \mathrm{~m}^{2} \mathrm{~s}^{-1}$ & - \\
\hline${ }^{b)}$ Diffusion coefficient $\mathrm{Na}^{+}$ & $D_{N a}$ & - & $0.65 \cdot 10^{-9} \mathrm{~m}^{2} \mathrm{~s}^{-1}$ \\
\hline Valence $\mathrm{Cl}^{-}$ & $z_{C l}$ & -1 & - \\
\hline Valence $\mathrm{Na}^{+}$ & $z_{N a}$ & - & +1 \\
\hline Initial concentration $\mathrm{Cl}^{-}$ & $C_{C l 0}$ & $5.25 \mathrm{M}$ & - \\
\hline Initial concentration $\mathrm{Na}^{+}$ & $C_{N a 0}$ & - & $1.33 \mathrm{M}$ \\
\hline Initial concentration fixed charges & $C_{f 0}$ & $5.25 \mathrm{M}$ & $1.33 \mathrm{M}$ \\
\hline${ }^{b)}$ Water attached to $\mathrm{Cl}^{-}$ & $\alpha_{C l}$ & $20 \%$ & - \\
\hline${ }^{b}$ Water attached to $\mathrm{Na}^{+}$ & $\alpha_{N a}$ & - & $5 \%$ \\
\hline b)Water attached to network & $\alpha_{f}$ & $80 \%$ & $95 \%$ \\
\hline Initial uniform swelling degree & $S D$ & 25 & 25 \\
\hline Initial water concentration & $C W_{0}$ & 93 vol. $\%$ & 89 vol. $\%$ \\
\hline
\end{tabular}

a) The value for $\mathrm{CN}$ corresponds to the regression shown in Figure 1c. Young's Modulus regression for VBS was not obtained. For this reason, the regression for $\mathrm{CN}$ was also used for VBS since Young's Modulus of both hydrogels are in the same range, and the influence of a small change in this parameter is not very significant for the predicted curvature. When the hydrogel is divided into two parts in the model, each part has its own Young's Modulus depending on its average swelling degree.

b) Estimated values.

Table S2. Zero-shear viscosity for different concentrations of sodium alginate solution and hydrogel pre-polymerized inks.

\begin{tabular}{lc}
\hline Sample & Viscosity, $\boldsymbol{\eta}$ (Pa.s) \\
\hline CN (ink) & $3.99 \pm 0.06 \cdot 10^{-3}$ \\
ALG $\left(25 \mathrm{mg} \cdot \mathrm{ml}^{-1}\right)$ & $5.54 \pm 0.27$ \\
ALG $\left(50 \mathrm{mg} \cdot \mathrm{ml}^{-1}\right)$ & $27.17 \pm 1.15$ \\
CN-ALG (ink, $\left.25 \mathrm{mg} \cdot \mathrm{ml}^{-1}\right)$ & $4.66 \pm 0.11$ \\
\hline
\end{tabular}


Movie S1. Voltage inversion test for $\mathrm{CN}$ hydrogel in an aqueous medium.

Movie S2. Voltage inversion test for $\mathrm{CN}$ hydrogel in ionic liquid.

Movie S3. CN hydrogel bending under ambient-air conditions.

Movie S4. CNG hydrogel bending under ambient-air conditions.

Movie S5. Gripper demonstrator holding a piece of paper.

Movie S6. 3D printing of a cube scaffold with CN-ALG ink. 


\section{References and Notes}

[1] R. Noe, W. Beck, M. Maase, A. Henne Germany, 2003.

[2] a) V. Leon, M. Quintana, M. A. Herrero, J. L. Fierro, A. de la Hoz, M. Prato, E. Vazquez, Chem. Commun. 2011, 47, 10936; b) J. M. Gonzalez-Dominguez, V. Leon, M. I. Lucio, M. Prato, E. Vazquez, Nat. Protoc. 2018, 13, 495.

[3] S. A. Dharaskar, K. L. Wasewar, M. N. Varma, D. Z. Shende, C. Yoo, Arab. J. Chem. 2016, $9,578$.

[4] S. Timoshenko, J. Opt. Soc. Am. 1925, 11, 233. 